\title{
Modelling the hydrodynamic effect of abrupt water depth changes on a ship travelling in restricted waters using CFD
}

\author{
Momchil Terziev $^{1 *}$, Tahsin Tezdogan ${ }^{1}$, Atilla Incecik $^{2}$ \\ ${ }^{1}$ University of Strathclyde, Department of Naval Architecture, Ocean and Marine \\ Engineering, Glasgow, UK. \\ ${ }^{2}$ University of Strathclyde, Faculty of Engineering, Glasgow, UK \\ *Corresponding author: momchil.terziev@strath.ac.uk
}

\begin{abstract}
Shallow water studies of ship hydrodynamics typically examine a single, constant underwater canal cross-section at a time. In practice, the underwater cross-sectional area and shape of the waterway is rarely, maintained constant over long distances. This study presents an attempt to quantify the effects of an abruptly varying water depth by numerically modelling such a condition using CFD. The results show that waves propagate and refract in the numerical towing tank in a physically consistent manner showing less than $0.1 \%$ error in the dissipation of a solitary wave when compared to analytical relations. A strong boundary layer is formed on the canal bottom almost as soon as the ship enters the shallower region. The resistance increase, resulting from the depth change is up to approximately $226 \%$ of the initial value near critical speeds.
\end{abstract}

Keywords: shallow water, restricted water, RANS, wavefield, step change in water depth, water depth transition

\section{Introduction}

Contemporary interest in the field of shallow water ship hydrodynamics is driven by the fact that according to EMSA (European Maritime Safety Agency, 2019, 2018, 2017, 2016, 2015), a large proportion of all ship incidents occur in restricted waters. Although human factors are predominantly thought to be the root cause of this, counter-intuitive ship behaviour can occur in shallow waters, magnifying the overall risk (Tuck, 1978). Therefore, understanding the hydrodynamic phenomena occurring in shallow, and more generally, restricted waters is of practical relevance.

In some cases, the ship operator may not be aware of the consequences caused by their operation in shallow water. An excellent example of this is described and analysed at length by Grue (2017), who investigated waves caused by ships sailing past abrupt depth transitions. He demonstrated that long waves can be generated at the depth transition, which the author termed "mini-tsunamis". The resulting waves were shown to cause substantial damage to coastal infrastructure several kilometres from their inception point. An even more extreme case is reported to have caused the loss of life (Soomere, 2007).

The present study takes inspiration form Grue's (2017) work and seeks to explore the associated effects further, using numerical methods. A survey of the literature revealed that studies model similar problems using potential-flow based methods. These generally fall within three categories including slender body methods (Gourlay, 2003; Plotkin, 1977, 1976; Tuck, 1967), 
Green function-based methods (Yang et al., 2001; Yuan, 2014; Yuan et al., 2018; Yuan and Incecik, 2016), methods based on the Boussinesq equation (Dam et al., 2008; David et al., 2017; Grue, 2017; Jiang et al., 2002; Torsvik et al., 2006; Wu and Wu, 1982), methods based on the Korteweg-de Vries equation (Cole, 1987; Hur, 2019; Katsis and Akylas, 1987, 1984), and methods based on the Kadomtsev-Petviashvili equation (Beji, 2018; Mathew and Akylas, 1990; Sharma, 1995).

Most methods mentioned previously can be thought of as long wave theories. The long wave family of theories can be arrived at by applying a combination of assumptions and appropriate boundary conditions to the Euler equations, which are known to model ship waves with adequate accuracy. However, as is often the case, there is some disparity between different approaches as illustrated by Torsvik (2009) in terms of dispersive properties exhibited by generated waves. Neglecting viscosity may not be a valid assumption, based on the findings of recent numerical and experimental studies, which observed the formation of a boundary layer on the seabed in very shallow conditions (Böttner et al., 2020; Shevchuk et al., 2016).

By contrast, studies have shown the fully nonlinear Reynolds averaged Navier-Stokes (RANS) equations can model the present class of problem well (Bechthold and Kastens, 2020; Elsherbiny et al., 2020; Shevchuk et al., 2016; Terziev et al., 2018; Tezdogan et al., 2016a). It is therefore prudent to attempt to construct a fully nonlinear viscous towing tank with a varying bathymetry. The specific object of the present study is thus to simulate the hydrodynamic effects caused by a ship passing over a step change in the water depth using CFD. To the best of the authors' knowledge, such towing tanks have only been constructed via the use of the family of long wave theories, described previously, and not with the aid of CFD. The novelty of this study therefore lies in the approach and problem adopted.

According to Jiang et al. (2002), the unsteadiness and three-dimensionality of the problem to be investigated herein precludes the use of many methods. Even the applicable methods rely on the assumption of inviscid flow, which may not hold for near-critical speeds or very shallow waters. Therefore, provided one can cope with the computational effort, it is desirable to investigate the effects of depth changes via a RANS method. Therefore, provided one can cope with the computational effort, it is desirable to investigate the effects of depth changes via a RANS method due to their inclusion of viscosity. The present work is the first to model this problem using the RANS approach.

This study presents an attempt at modelling the above scenario using the commercial RANS solver Star-CCM+, version 14.06. As a starting point, the experimentally investigated rectangular canal of Elsherbiny et al. (2019) is used. This features a depth-to-draught ratio of 2.2 and a width of $4.6 \mathrm{~m}$. To examine phenomena other methods may not be well-suited for as the chosen speed range is trans-critical. Specifically, two subcritical $\left(F_{h}=U / \sqrt{h g}<1\right.$, where $U$ is the ship speed, $g$ is the gravitational acceleration, and $h$ is the water depth) depth Froude numbers are chosen to begin with. As the ship advances through the domain, it encounters a step increase in the water depth, rendering a higher value of $F_{h}$. To model the motion of the ship, use is made of the overset domain approach, following recent work by the authors (Terziev et al., 2020).

The specific cases examined in this study are detailed in the following section. Section 3 presents the numerical implementation, which also contains estimates for the numerical 
uncertainty. Then, the generated results and their analysis are given in Section 4. Finally, Section 5 summarises the study reported in this paper and suggests future research topics.

\section{Case studies}

This section is devoted to an overview of this work's selected case studies. To begin with, justification is given in terms of the selected conditions.

As stated previously, transitions past the critical depth Froude number are sought. This is to demonstrate that RANS solvers are well-equipped to handle such problems, that present significant difficulties for several theoretical methods as reported in the literature. For example, the slender body theory has a singularity at $F_{h}=1$. Several studies have devised approaches to handle the behaviour of the theory around this depth Froude number, but none has become widely used (Alam and Mei, 2008; Gourlay and Tuck, 2001; Lea and Feldman, 1972; Miles, 1986; Tuck, 1967, 1966). Therefore, the decision to simulate conditions where the critical speed is met and exceeded was taken. Alongside these, subcritical conditions are also modelled.

To achieve a high depth Froude number, a relatively high ship speed is required. Although it may be the case that few vessels would operate under such conditions, Grue (2017) reported fast ferries travelling at $F_{h}=0.7$ prior to the depth transition. Therefore, the high-speed choice is deemed both acceptable and practically relevant for ship operations. Since ship waves are of greater concern in restricted waterways due to bank erosion, such as rivers and canals, a corresponding case study is sought.

As mentioned earlier, the work of Elsherbiny et al. (2019) is used in this study, as a starting point for three reasons. Firstly, they investigated a canal case, which matches what is required. Secondly, the present authors have already laid the groundwork in constructing and validating the wavefield of this case study in recent work (Terziev et al., 2020). Finally, replicating the towing conditions allows other researchers to compare resistance and wavefield data against the work presented herein. This could be done by other researchers using numerical methods. Alternatively, an experimental version of the case studies presented here should also be carried out.

Initial speeds are selected based on the highest available $F_{h}$ explored by Elsherbiny et al. (2019), namely, $F_{h i}=0.77$. Since one of the objectives of this study is to model a critical case, we restrict the water depth so that when the ship crosses the step at a speed, corresponding to $F_{h s}=1$. Henceforth, the subscripts $i$ and $s$ will be used to denote the initial condition, and the condition past the step, respectively. Naturally the same ship is used as in Elsherbiny et al. (2019), the KCS, whose principal characteristics are shown in Table 1.

Table 1. KCS principal characteristics (in model scale).

\begin{tabular}{|l|l|l|l|}
\hline Quantity & Symbol & Value & Unit \\
\hline
\end{tabular}




\begin{tabular}{|l|l|c|c|} 
Scale factor & $\lambda$ & 75 & - \\
\hline Length & $L$ & 3.067 & $\mathrm{~m}$ \\
\hline Beam & $B$ & 0.429 & $\mathrm{~m}$ \\
\hline Draught & $T$ & 0.144 & $\mathrm{~m}$ \\
\hline Block coefficient & $C_{B}$ & 0.651 & - \\
\hline Longitudinal Centre of Gravity & $L C G$ & 1.488 & $\mathrm{~m}$ \\
\hline Wetted area & $S$ & 1.694 & $\mathrm{~m}^{2}$ \\
\hline
\end{tabular}

Making the above choice while maintaining a constant velocity requires the depth to change from $h_{i} / T=2.2$ to $h_{s} / T \approx 1.3$ (note that the width of the canal, $w=4.6 \mathrm{~m}$, is maintained constant). Further cases to gauge the sensitivity of the flow to water depth are specified at three equal intervals between the two extremities in terms depth Froude number. Moreover, to include additional supercritical cases, a second initial depth Froude number is selected as $F_{h i}=$ 0.9 and ran on the same depths. The resulting test matrix is given in Table 2. Further reductions in the water depth are not implemented to avoid numerical problems in the implementation of the overset domain approach, used to tow the ship. A schematic drawing of the different steps investigated can be seen in Figure 1.

Table 2. Text matrix.

\begin{tabular}{|c|c|c|c|c|c|c|c|c|}
\hline No & $U(\mathrm{~m} / \mathrm{s})$ & $F_{h i}$ & $h_{i}(\mathrm{~m})$ & $h_{i} / T$ & $F_{h s}$ & $h_{s}(\mathrm{~m})$ & $h_{s} / T$ & $h_{i} / h_{s}$ \\
\hline 1 & \multirow{4}{*}{1.364} & \multirow{4}{*}{0.77} & \multirow{8}{*}{0.32} & \multirow{8}{*}{2.2} & 1 & 0.190 & 1.304 & 1.687 \\
\hline 2 & & & & & 0.943 & 0.214 & 1.468 & 1.500 \\
\hline 3 & & & & & 0.885 & 0.242 & 1.665 & 1.321 \\
\hline 4 & & & & & 0.826 & 0.277 & 1.905 & 1.155 \\
\hline 5 & \multirow{4}{*}{1.595} & \multirow{4}{*}{0.9} & & & 1.169 & 0.190 & 1.304 & 1.687 \\
\hline 6 & & & & & 1.102 & 0.214 & 1.468 & 1.500 \\
\hline 7 & & & & & 1.034 & 0.242 & 1.665 & 1.321 \\
\hline 8 & & & & & 0.967 & 0.277 & 1.905 & 1.155 \\
\hline
\end{tabular}

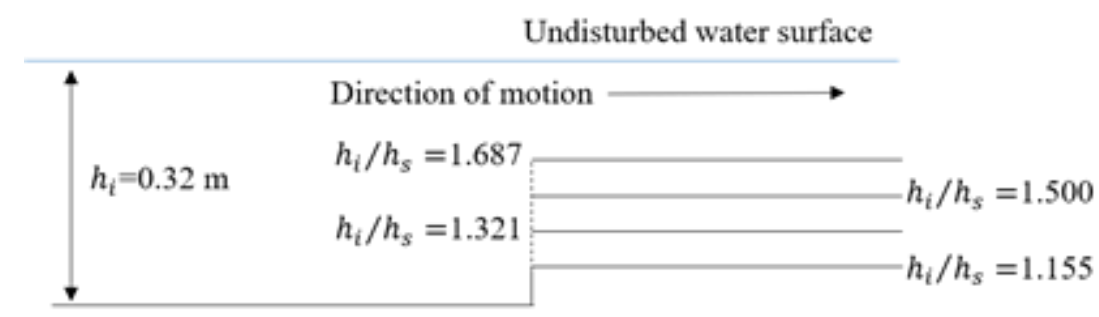

Figure 1. Schematic drawing of the step changes in water depth. Not drawn to scale.

\section{Numerical implementation}

This section is devoted to the numerical implementation, with subsections assigned to different aspects of the simulations.

\subsection{The numerical environment}

As stated in the introduction, the commercial solver, Star-CCM+, version 14.06 is used in this study. The solver is based on the Finite Volume Method (FVM). To avoid deviating from the 
core topic of this work, specific details in terms of algorithms and subroutines are not discussed. Instead, the reader is referred to the user manual (Siemens, 2018) and texts containing detailed information of the numerics used (Ferziger and Peric, 2002).

There are two main aspects of the solution in the present CFD modelling requiring particular attention. The first relates to the definition of the free surface. The disturbance caused by the ship may be significant and will influence the solution substantially. Therefore, an accurate representation is necessary. The definition of the water surface is modelled via the Volume of Fluid (VoF) method (Hirt and Nichols, 1981), with the High Resolution Interphase Capturing (HRIC) scheme to enhance its sharpness (Muzaferija and Peric, 1999, 1997). The grid on which the problem at hand is discretised is discussed in the following subsections.

The second aspect of the numerical implementation that is of importance relates to turbulence. In this study, the $k-\omega$ model of Wilcox (2008) is used. The $k-\omega$ model provides consistently good predictions in terms of computational resources and resistance predictions, as demonstrated in recent studies (Eca and Hoekstra, 2008; Elsherbiny et al., 2020). The twoequation eddy-viscosity turbulence closure is also selected due to its seamless application to all types of meshes. This is an advantage because the model does not require modifications depending on whether a wall function is used or not. Although a low $y^{+}$mesh is constructed on the ship hull, as will be demonstrated subsequently, this is not the case for the canal sides and bottom. Therefore, wall functions are used at all domain boundaries. To ensure a good representation of turbulent properties, all simulations are run with a second order accurate convection scheme.

To model the ship's longitudinal motion along the domain, the overset domain approach is used. In essence, this results in the creation of a box, enveloping the hull. To re-create the ship's motion in the $x$ direction, the overset domain is assigned the ship's velocity, which can be consulted in Table 2. Adopting the overset domain allows the efficient modelling of the problem at hand. The alternative would involve re-meshing at each time-step, which would cause considerable computational overheads.

\subsection{Computational domain and boundary conditions}

The computational domain dimensions and arrangement are depicted in Figure 2. The location labelled as "Step" represents where the water depth transition is located. The values used in this study can be consulted in Table 2 and Figure 1.

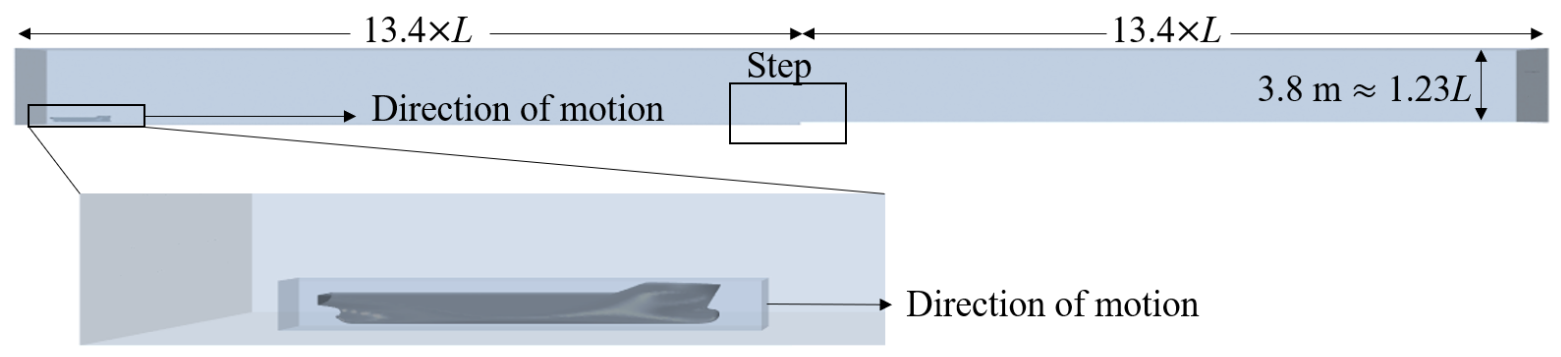

Figure 2. Depiction of the computational domain (depicted: $h_{i} / h_{s}=1.687$ ).

The main dimensions of the domain are unaltered across case studies. The only change stems from the difference in the water depth after the midpoint of the domain. This is also set as the global origin to simplify the representation of the results. To further simplify the results, the 
ship is modelled with an even keel. In other words, ship squat is not accounted for. The reason behind this relates to the restricted water depth. If the overset domain were to collide with the background domain, the simulation may fail or cause unreasonable results. Since a shock is expected as the ship transits past the step, ship squat is not modelled to avoid the aforementioned effects.

The modelling of the KCS with an even keel results in a substantially different resistance values to what is recorded in Elsherbiny et al. (2019), precluding the possibility of a validation study. However, using an identical set-up, the present authors validated both the resistance and wavefield in their several past studies published in different scientific journals (Elsherbiny et al., 2019a; Terziev et al., 2019, 2020; Tezdogan et al., 2015, 2016b).

The manner in which the numerical towing tank is constructed allows the removal of all open boundary conditions. This carries positive and negative impacts on the solution simultaneously. Specifically, the modelling assumptions related to inlets and outlets, damping lengths and clearance between an open boundary and the ship, inlet turbulence, etc. are no longer of consequence because they no longer exist in the this work's CFD simulation. Therefore, the modelling assumptions and sources of uncertainty are significantly reduced. On the other hand, from a mathematical point of view, open boundaries are easier to implement. Their removal may destabilise the solution in some cases. This is particularly the case when performing grid refinement studies - if the grid is too coarse, the simulation diverges during the early stages of the solution.

The numerical implementation of the domain results in three types of boundary conditions. Symmetry planes are instituted in the overset and background domains, coincident with the centreline to reduce the computational effort. The overset domain requires the appropriate boundaries imposed on the moving box, encasing the ship. All other boundaries are set as noslip walls, as would be the case in a physical towing tank. Therefore, the numerical tank is physically consistent with physical towing facilities.

\subsection{Computational mesh}

The computational mesh onto which the RANS equations are discretised is generated within the automatic facilities of the software package used. To ensure a good representation of the water surface, the mesh used in a recent study where a Fourier technique was used to validate the results, is replicated (Terziev et al., 2020). The prism layer mesher, offered by Star-CCM+ is used to create near-wall cells at the ship hull, with the average y+ not exceeding 0.8 for the highest speed examined. This is used to construct the near-wall cells on the ship hull, responsible for accounting for the high velocity gradients within the boundary layer. On the other hand, the $y+$ values on the side walls and bottom are allowed to exceed 1, resulting in the use of wall functions on these boundaries. A close-up of the generated grid on the undisturbed free surface prior to initiating the simulation is shown in Figure 3. 


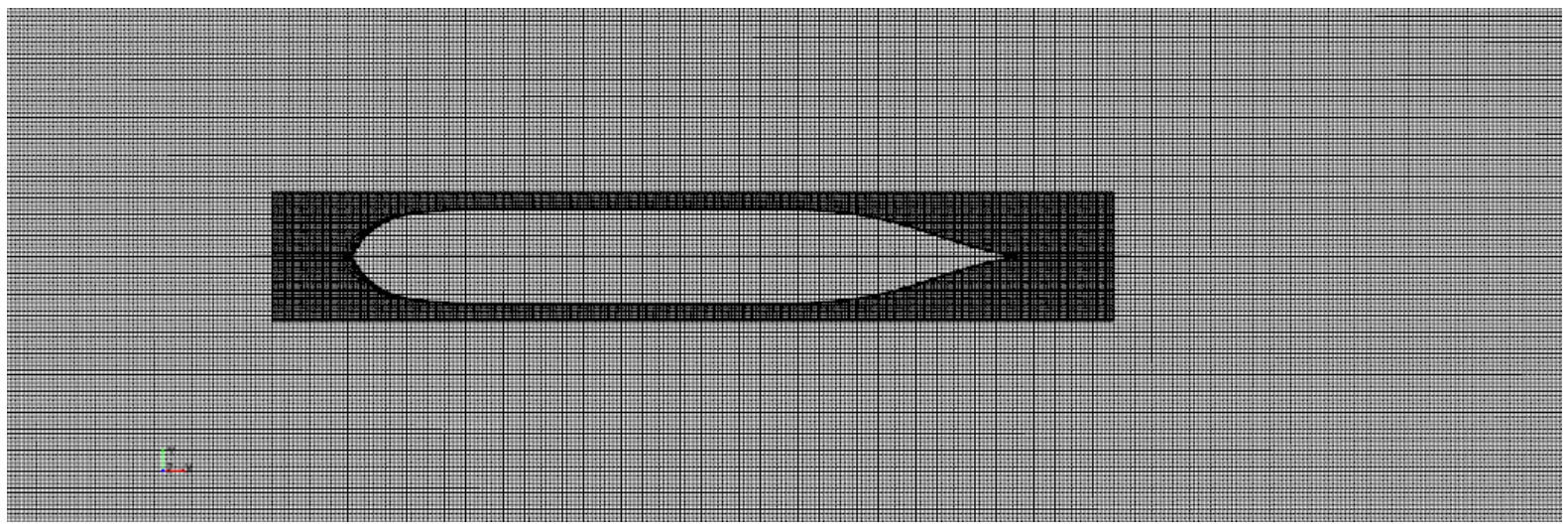

Figure 3. Close-up of the computational mesh on the free surface.

Table 3 contains the numbers of cells, generated for each case. It should be noted that these do not vary with $F_{h i}$. Therefore, both $F_{h i}=0.77,0.9$ are ran with the same numbers of cells for their corresponding cases.

Table 3. Cell numbers for all four depth transitions

\begin{tabular}{|l|l|r|}
\hline Cases & $h_{i} / h_{s}$ & Number of cells \\
\hline 1 and 5 & 1.687 & $25,248,501$ \\
\hline 2 and 6 & 1.500 & $25,632,314$ \\
\hline 3 and 7 & 1.321 & $26,392,544$ \\
\hline 4 and 8 & 1.155 & $26,776,892$ \\
\hline
\end{tabular}

\subsection{Time-step selection}

Making an adequate choice for the time-step $(\Delta t)$ in unsteady simulations is of critical importance. If the $\Delta t$ value is too large, the numerical solution may become unstable, or give unrealistic results. This is to be balanced with computational time - it is not practical for a simulation to run over unnecessary long periods of time while consuming computational resources. Numerical noise may also manifest itself in the solution if the time-step is not chosen correctly. Based on the results of Terziev et al. (2020), $\Delta t=0.0035 \mathrm{~L} / U$ is chosen with a first order discretisation scheme. To ensure adequate representation of the physics, the Courant number is monitored throughout the simulation, with a stopping criterion imposed to end the simulation if the Courant number equals or exceeds one within the overset domain.

The overset domain is chosen instead of the background to represent the Courant number criteria because in the former, the majority of the flow is accelerated. Had this been applied to the background domain, where the majority of the fluid is static, the result in terms of the Courant number would be misleadingly low. The generated grid succeeded in preserving a Courant number, CFL $<1$.

\subsection{Numerical verification}

This subsection contains estimates of the numerical uncertainty, induced by the discretisation of the RANS equations in time and space. The approach used follows the recent work of Bechthold and Kastens (2020), who followed the guide of Celik et al. (2008). This begins with the definition of a refinement factor, $r$. According to ASME (American Society of Mechanical Engineers, 2009), acceptable values of $r$ range between 1.1 and 1.5. In the present study, the value of $\sqrt{2}$ is adopted. This is applied as a multiplicative factor to the mesh and time-step, 
which are magnified successively, creating a medium $(i=2)$ and coarse $(i=3)$ solution for each metric (mesh and time-step). To simplify the analysis, the refinement ratio is kept constant, i.e. $r_{21}=r_{32}=\sqrt{2}$. Nevertheless, the relationships used to perform the analysis with non-uniform $r$ are adopted to enable comparison of results with other studies.

Once the medium and coarse solutions have been obtained, the observed order of accuracy can be determined as shown in Eq. (1):

$p=\frac{|\ln | \epsilon_{32} / \epsilon_{21}|+q(p)|}{\ln \left(r_{21}\right)}$,

with

$q(p)=\ln \left(\frac{r_{21}^{p}-s}{r_{32}^{p}-s}\right)$

and

$s=\operatorname{sgn}\left(\frac{\varepsilon_{32}}{\varepsilon_{21}}\right)$,

where $\varepsilon_{32}=\phi_{3}-\phi_{2}$, and $\varepsilon_{21}=\phi_{2}-\phi_{1}$, with $\phi_{i}$ denoting the $i^{\text {th }}$ solution. For a constant refinement case, the function $q(p)=0$. In the case of mesh independence, the medium and coarse solution featured 8,684,955 and 3,167,970 cells, respectively.

The next step is to estimate the uncertainty, denoted GCI (Grid Convergence Index), after Roache (1998), shown in Eq. (4):

$G C I=1.25 \times\left|\frac{\phi_{1}-\phi_{2}}{\phi_{1}}\right| /\left(r_{21}^{p}-1\right)$

In the present case, the resistance of the ship before and after the step change in the depth is used in the assessment. Therefore, two different estimates of the uncertainty are obtained for the mesh and two for the time-step. The specific case to which this is applied is case $1, F_{h i}=$ $0.77, F_{h s}=1$, as shown in Table 4 .

Table 4. Numerical uncertainty study results (results are given for the ship resistance).

\begin{tabular}{|l|r|r|r|r|}
\hline & \multicolumn{2}{|c|}{ Before step $\left(F_{h i}=0.77\right)$} & \multicolumn{2}{c|}{ After step $\left(F_{h s}=1\right)$} \\
\hline & Mesh & \multicolumn{1}{c|}{ Time-step } & \multicolumn{1}{l|}{ Mesh } & Time-step \\
\hline Fine (N) & 19.74 & 19.74 & 64.43 & 64.43 \\
\hline Medium (N) & 23.09 & 23.05 & 53.40 & 65.76 \\
\hline Coarse (N) & 23.85 & 23.04 & 47.64 & 65.76 \\
\hline$G C I$ & $6.20 \%$ & $0.03 \%$ & $10.94 \%$ & $0.01 \%$ \\
\hline$U_{c}$ & \multicolumn{3}{|c|}{$0.06 \%$} & \multicolumn{2}{c|}{$0.11 \%$} \\
\hline
\end{tabular}

In Table 4, the GCI value before and after the step is reported. Then, the combined uncertainty, $U_{c}$ is estimated for each case as shown in Eq. (5):

$U_{c}=\sqrt{G C I_{\text {mesh }}^{2}+G C I_{\text {time }}^{2}}$

It is apparent from the results of Table 4 that the results are more sensitive to variations in the mesh than they are to the time-step. Although the uncertainty exceeds $10 \%$ for the mesh in the 
critical region, this is considered a tolerable level of uncertainty considering the challenging case to which the analysis is applied to. The results from this subsection can be interpreted as follows. The mesh requirements for critical depth Froude number cases are considerably higher than those for subcritical cases. This serves to highlight that the examination of critical speeds is not a trivial problem even for RANS solvers. However, results with the given uncertainty can be obtained, whereas many potential flow-based methods predict singularities at $F_{h}=1$.

Finally, it should be noted that while coarsening the grid, the finest time-step was maintained. The mesh was magnified by the same factor $(r=\sqrt{2})$ in both the overset and background domain to preserve the transitional ratio between the two. Conversely, the temporal dependence study was carried out on the finest mesh only.

\section{Results and discussion}

This section presents the obtained results, their analysis and discussion. To begin with, the resistance characteristics of the ship are presented. For convenience, the percentage increase for the model-scale ship in resistance as the water depth changes is presented. This is thought to be a more suitable way to enable other researchers to compare different hull forms subjected to similar conditions. The initial resistance recorded for the model-scale ship at $F_{h i}=0.77$ was $19.74 \mathrm{~N}$, while at $F_{h i}=0.9$ the value was $55.891 \mathrm{~N}$.

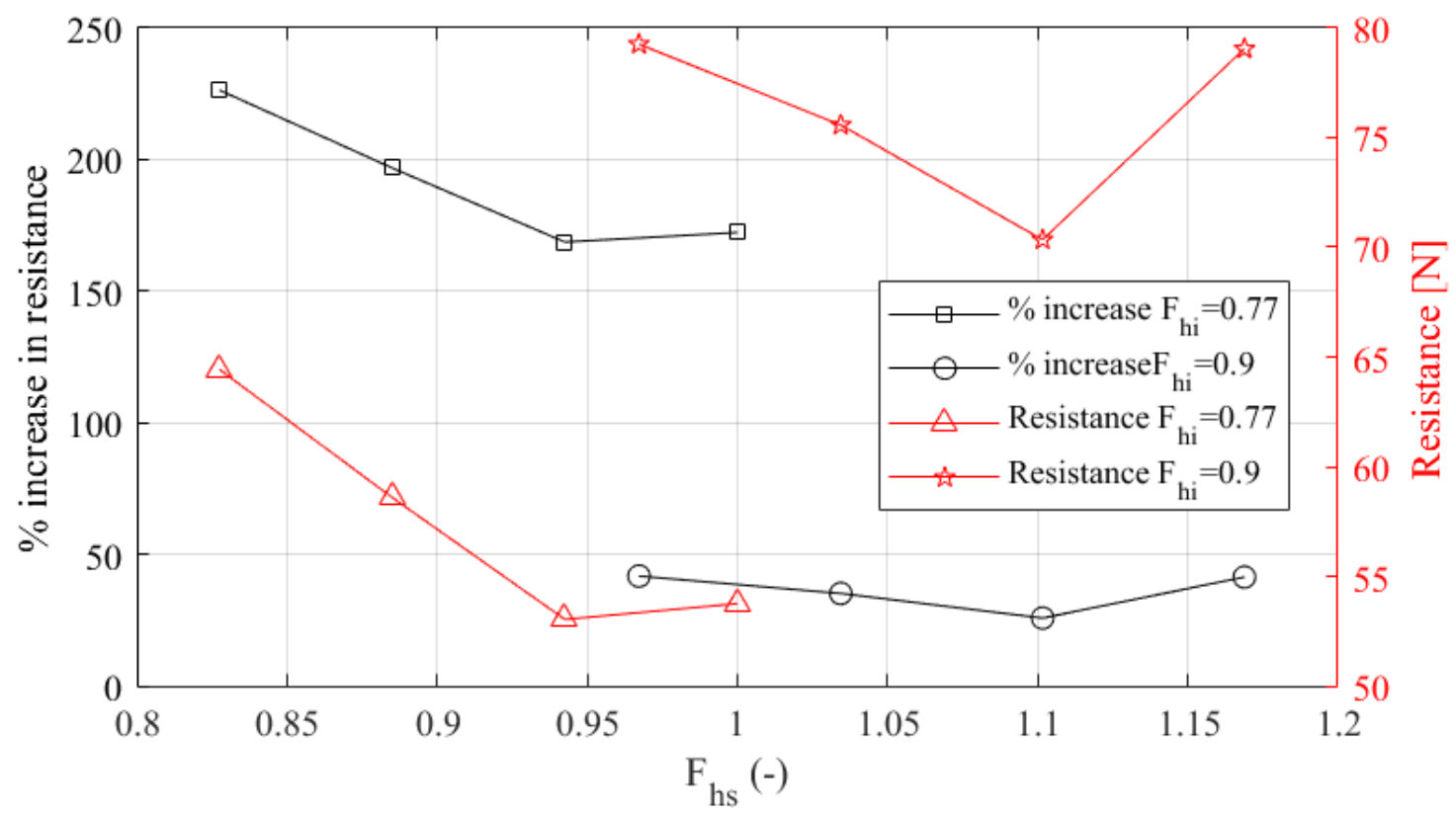

Figure 4. Resistance increase resulting from the depth change.

Figure 4 demonstrates that the initial velocity is critically important for the relative increase in resistance. To elaborate, the cases where the initial speed is $F_{h i}=0.77$ exhibit several times the increase in resistance when $F_{h i}=0.9$. More importantly, no jumps or sharp cusps are observed, as predicted by linear potential flow theory. The results in Figure 4 also point towards the possibility to minimise the total drag by small variations in the ship speed. For example, case $3\left(F_{h s}=0.885\right)$ shows a considerably smaller increase in resistance than case $1\left(F_{h s}=1\right)$.For both initial depth Froude numbers, the resistance does not peak at $F_{h}=1$. This is in line with experimental data for a family of hulls given in Benham et al. (2020) and Benham et al. (2019), where the authors predicted that wave resistance peaks well before the critical depth Froude 
number. The reason why ship resistance is thought to exhibit a peak near $F_{h}=1$ is strictly related to the sustained generation of waves, which is widely considered an independent component of the total resistance. Linear potential flow theories place the peak at the critical depth Froude number; however, nonlinear phenomena are known to deform the wave resistance curve's peak towards the lower $F_{h}$ range. A similar effect can be observed in other experimental data in terms of the Kelvin wake angle $(\theta)$. For example, Johnson's (1957) experiments showed that the peak in $\theta$ can occur at around $F_{h, \theta=\max }=0.9$. Therefore, the trend exhibited by both curves in Figure 4 matches expectations.

In the cases where $F_{h s} \geq 1.1$, one might expect to observe a reduction in the resistance. This is a well-known phenomenon and has recently been demonstrated by Benham et al. (2020, 2019). There are two possible explanations as to why this is not observed in the presented results, which suggest the resistance increases in each case. One way to look at the data would be to suggest that the expected decrease in resistance is too narrow over the depth Froude number range. In such a scenario, the dip would be observed if further simulations were carried out between $1.09<F_{h s}<1.15$. Evidence to suggest that this may be the case can be found in Benham et al. (2019). The decrease in resistance for their hull forms is narrow. It is also worth noting that in their study, a family of simple shapes were examined. Similar hulls, for example, the Wigley hull, are known to produce a predominantly linear flow field (Chen et al., 2016).

A second way of interpreting these results may be in terms of the wavefield. When the depth Froude number past the step exceeds or is equal to unity, any solitons generated by the ship may not be shed sufficiently quickly forward. Therefore, the ship may be trapped sailing against a wave. One way to determine whether this is the case would be to examine the wave field. This is shown in

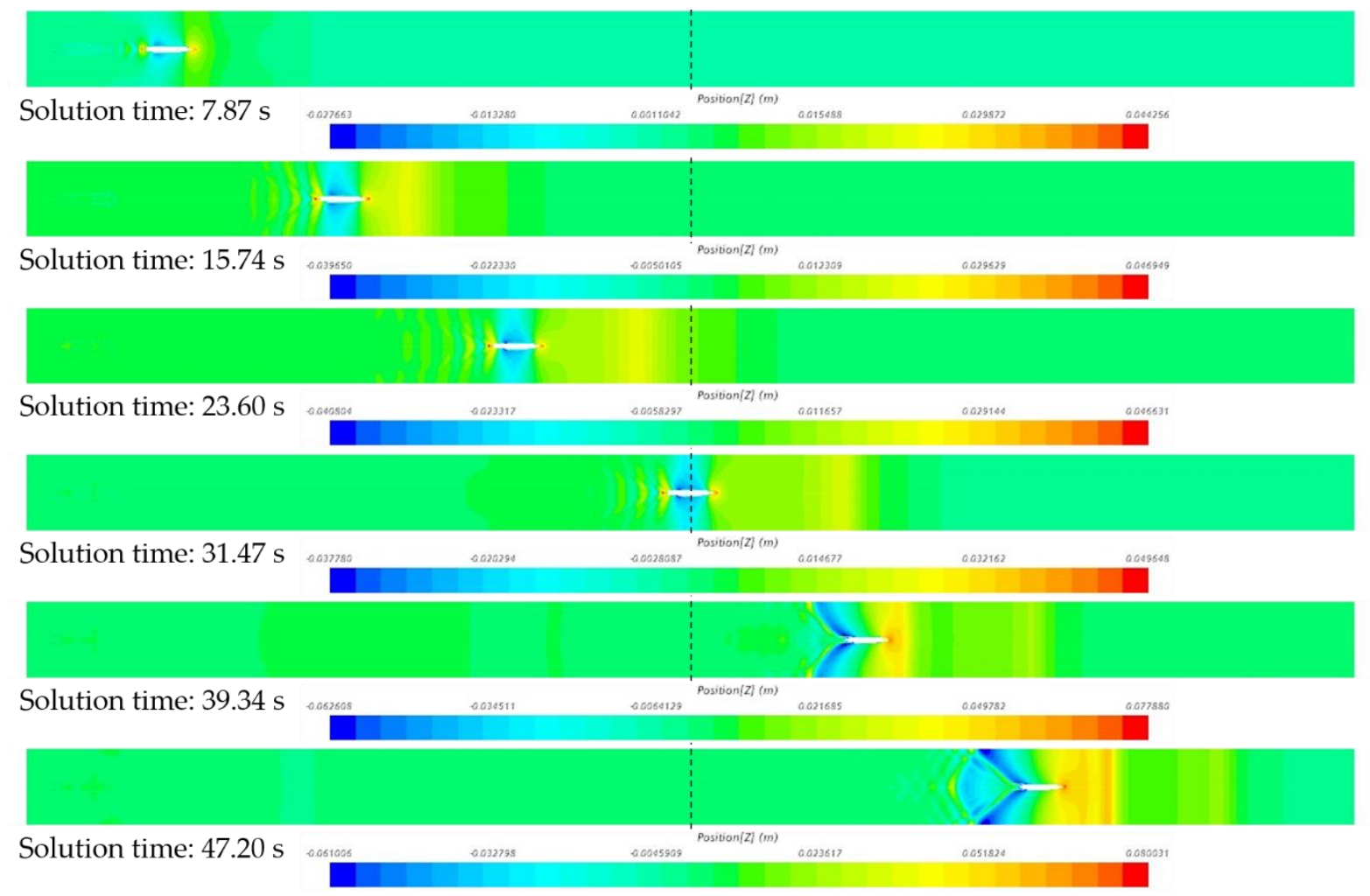

Figure 


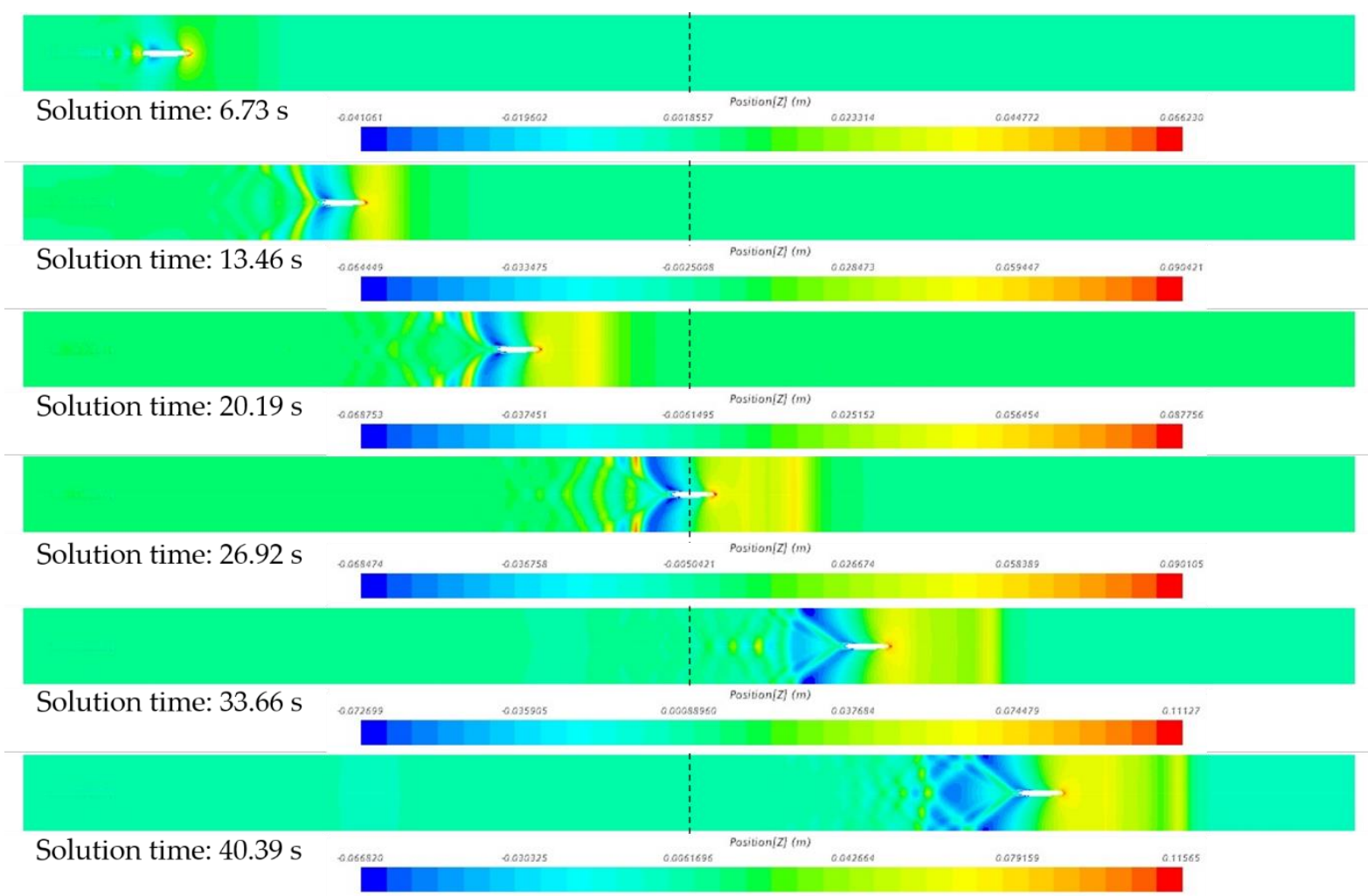

Figure 6 for cases 1 and 7, respectively $\left(F_{h i}=0.77\right.$ with $F_{h s}=1$, and $F_{h i}=0.9$ with $F_{h s}=$ 1.03 , respectively).

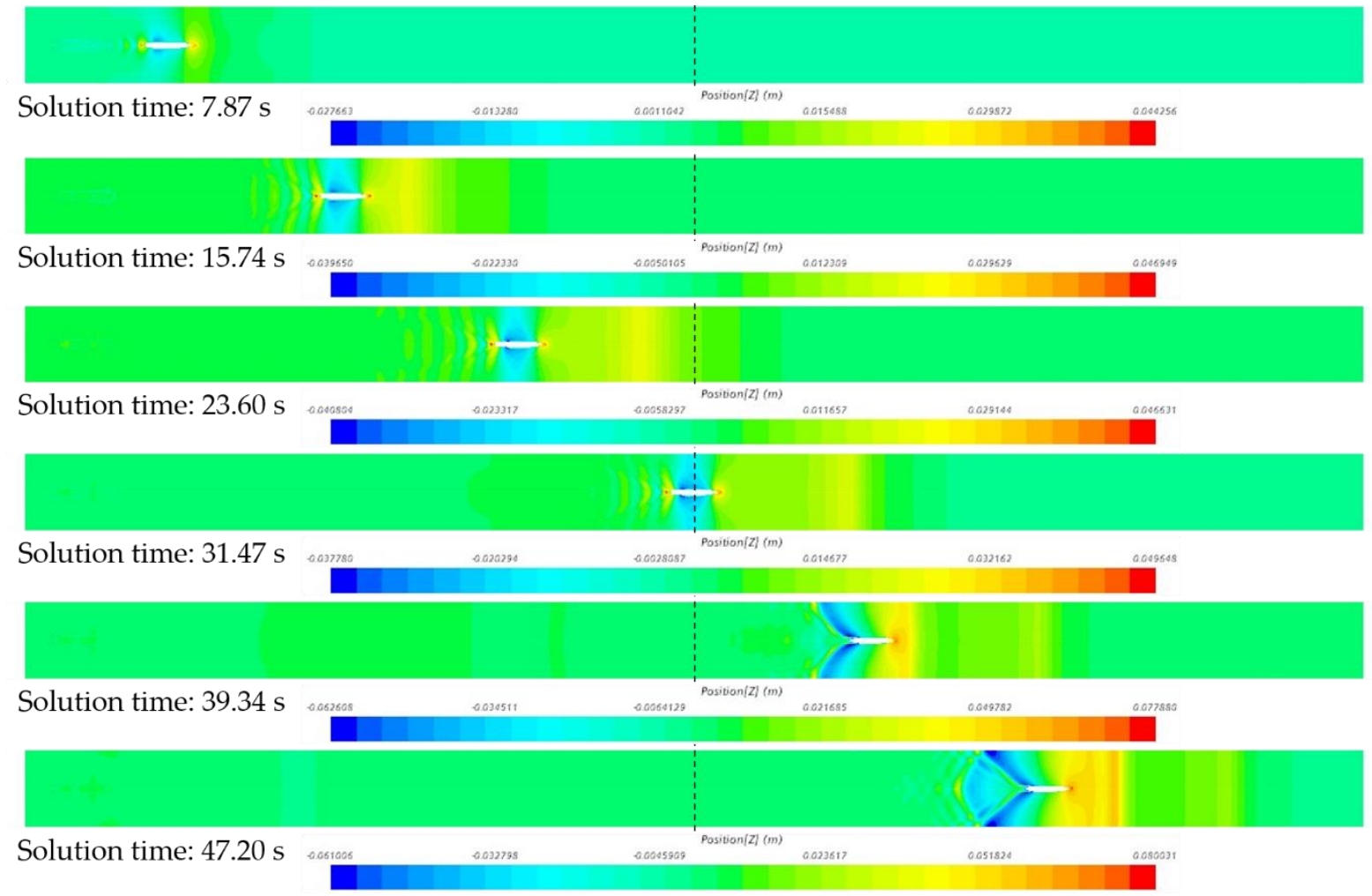

Figure 5. Wavefield for case $1, F_{h i}=0.77$ and $F_{h s}=1$. The solution time and increment interval unit at which the free surface is shown is based on the end of the acceleration phase 
(shown in the first tile). The dashed line indicates the position of the step change in water depth.

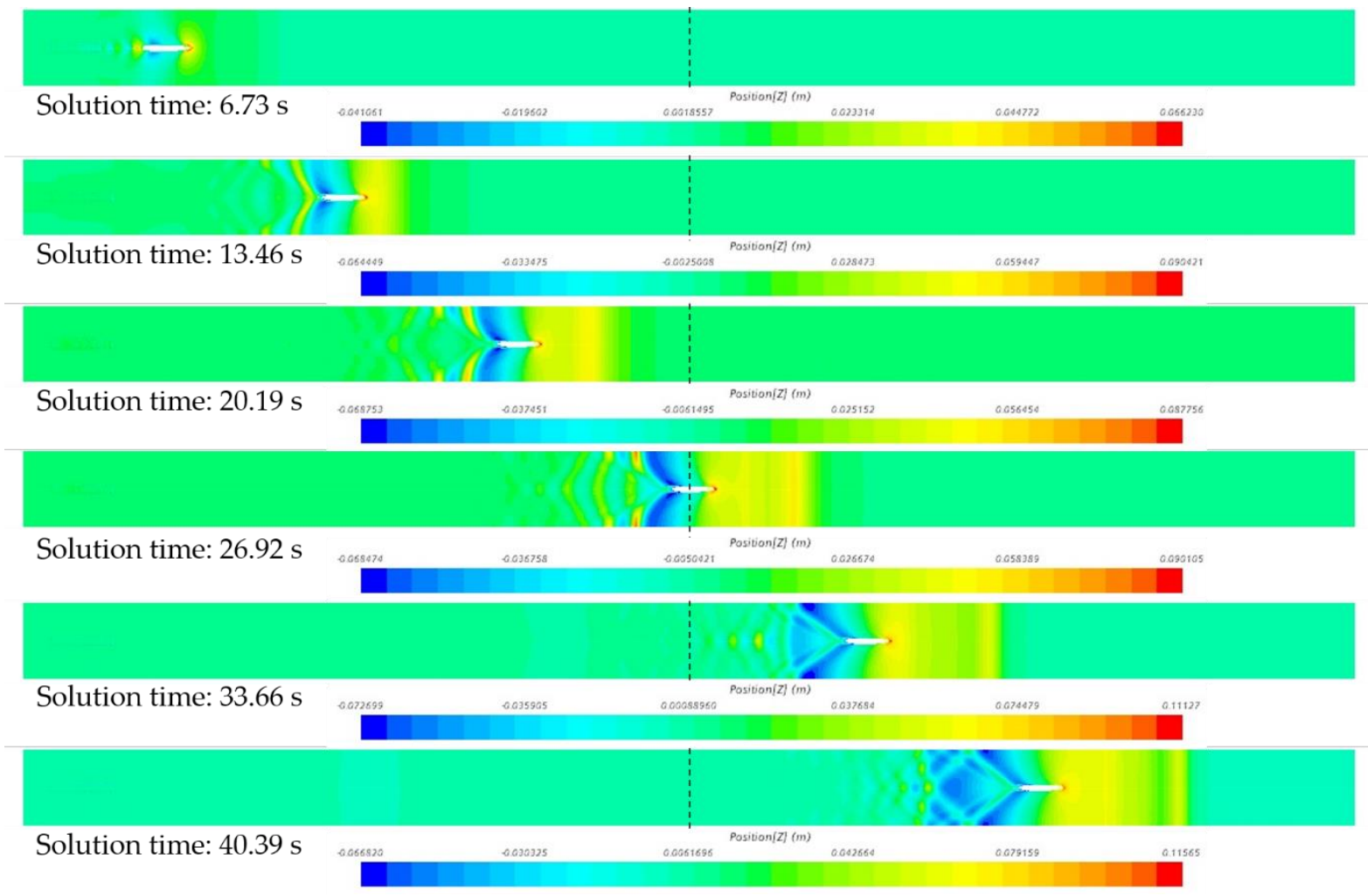

Figure 6. Wavefield for case $7, \mathrm{~F}_{\mathrm{hi}}=0.9$ and $\mathrm{F}_{\mathrm{hs}}=1.03$. The solution time and interval at which the free surface is shown is based on the end of the acceleration phase (shown in the first tile). The dashed line indicates the position of the step change in water depth

The wave elevation carried in front of the ship is longer for $F_{h i}=0.9$ and $F_{h s}=1.03$ (case 7). Thus, it is conceivable that this is could be the root cause for the elevated resistance. The second main difference between 


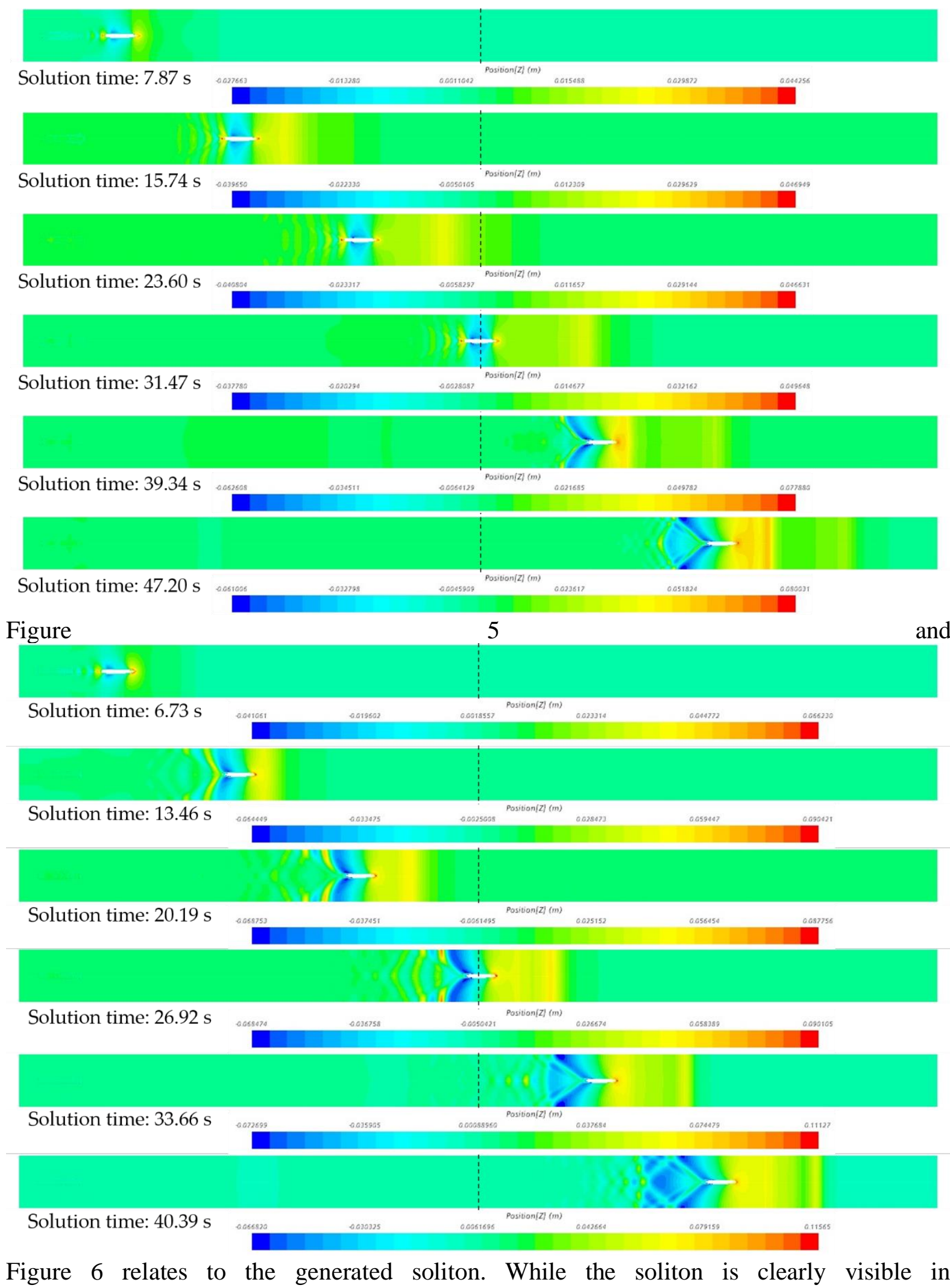




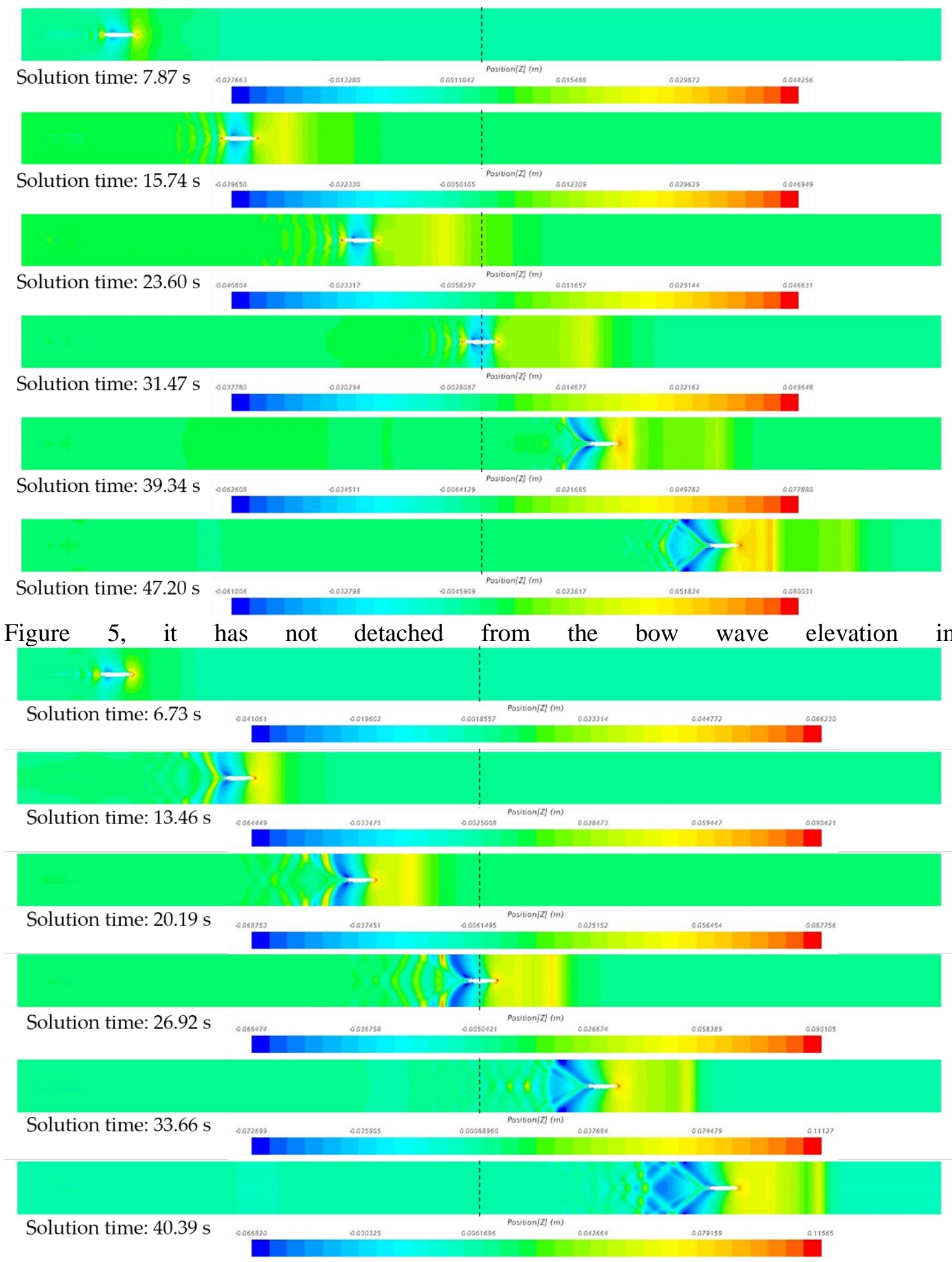

Figure 6. This is the case because the ship speed is close to the wave speed $\left(F_{h i}=0.9\right)$, therefore, the soliton would require a much longer domain to be properly shed. Solitons are also known to be essentially two-dimensional (Gourlay, 2001). To check whether the wavefield is $2 \mathrm{D}$ on the step, the time-history of the wave elevation on the step is recorded and shown in Figure 7. The free surface is monitored at four points next to the ship to provide a picture of the generated disturbance with distance in the $y$ direction. Since the towing tank is symmetrical about the centreline, the probes are not mirrored. For comparison, the time-history of the same 
probes shown in Figure 7 for $F_{h i}=0.77$, are shown for $F_{h s}=0.9$ in Figure 8 . These demonstrate that the wavefield in the latter case is $2 \mathrm{D}$ to a much greater extent and that the change in step height $\left(h_{s}\right)$ has a smaller relative influence on the deformations of the free surface.
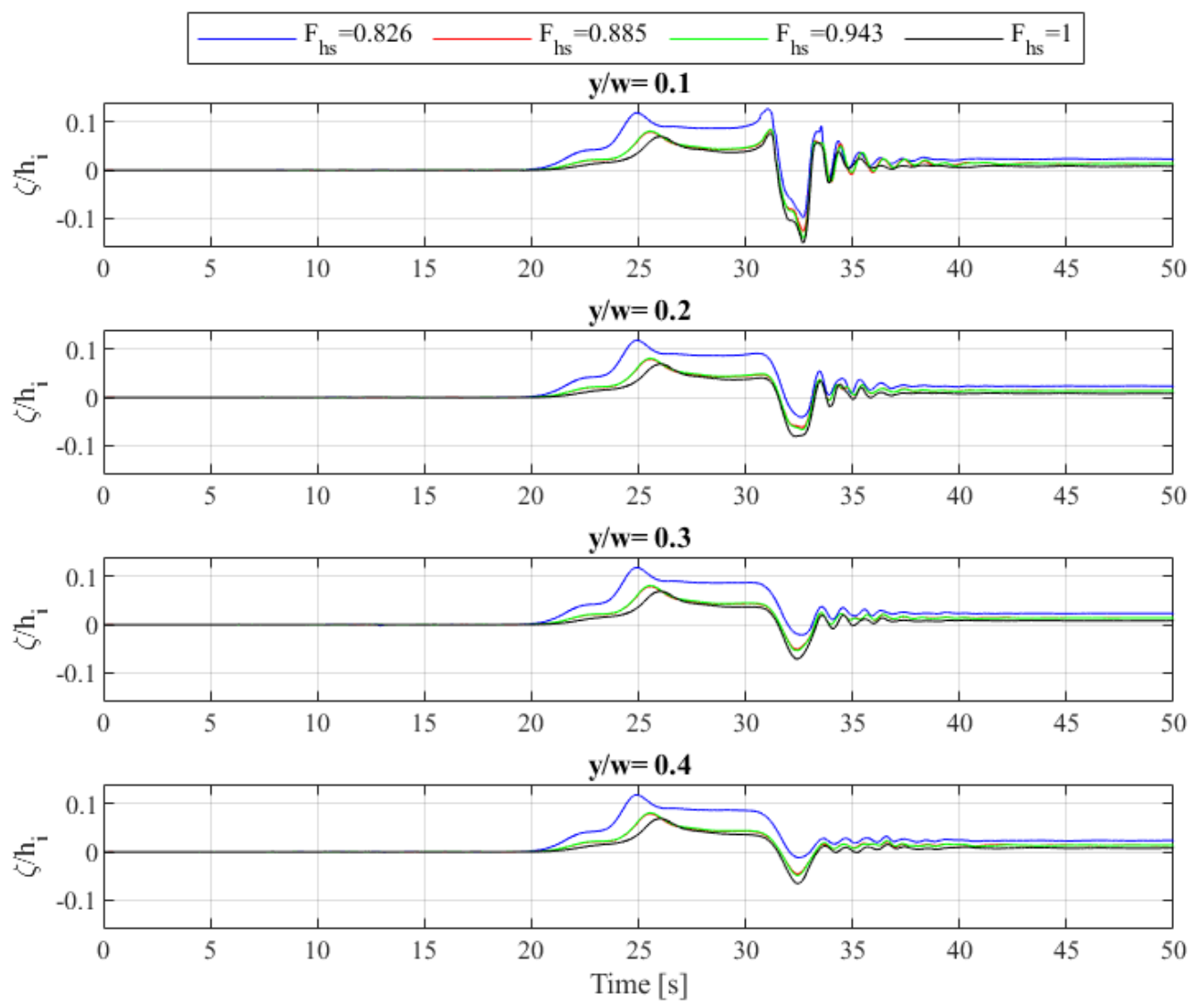

Figure 7. Wave probes at the step for $F_{h i}=0.77$. 

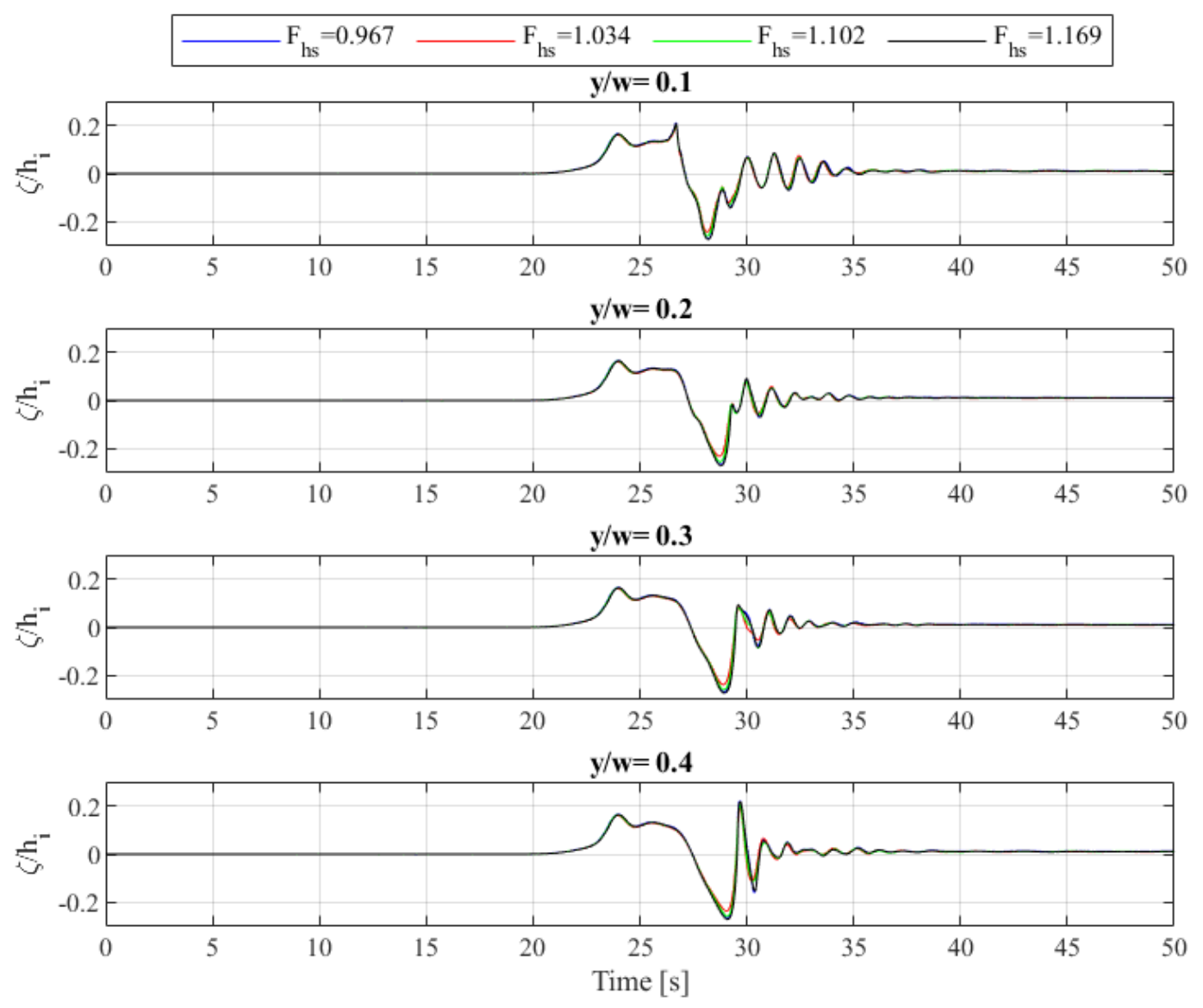

Figure 8. Wave probes at the step for $F_{h i}=0.9$.

Figure 7 indicates that the wave field is only uniform along the $y$ axis prior to the ship's interaction with the step. This can be seen by referring to the wave elevation between 20 and 30 seconds of physical time in Figure 7 and Figure 8 . The interactions differ with $F_{h s}$ due to the physics of wave reflection and transition from a submerged step. Since the wave speed in the deeper region is higher than that of the shallower region, past the step, the wave profile must transform upon transiting from one depth to the other. There have been many studies into how this occurs. The first such work is typically attributed to Lamb (1932), who derived an expression for the ratio of transmitted and incident wave. His assumption of zero vertical velocity at the step seemed inappropriate to Bartholomeusz (1958), who presented a more indepth study. However, the end result was identical to Lamb's (1932). Later, both of these studies were put under question by Newman (1965), who also ended up with Lamb's (1932) formulation for very shallow water cases.

The contribution of Newman (1965) however was expressed in the fact that he obtained an expression for an infinitely deep incident wave transforming into a shallow region. He provided a physical interpretation as to why a transmitted wave asymptotically tends to a wave height $\zeta_{s}=2 \times \zeta_{i}$ (where $\zeta$ is the wave elevation, whereas the subscripts maintain their earlier designation) as $h_{s} \rightarrow 0$. Newman's (1965) interpretation is that as the shallower region's depth vanishes, two phenomena occur. Firstly, the entire incident wave's amplitude is reflected, which is physically consistent. Secondly, that as the wave transits to much shallower regions, the energy transmitted into the region of depth $h_{s}$ reduces at a rate proportional to the $h_{s}$, causing the transmitted component to be twice the incident wave's height. Newman (1965) 
then presents experimental results, which show that the theory is consistent, although some scatter in the tank data is observed around the theory. To check whether the physical phenomena occur in agreement with the aforementioned studies, the wave elevation is recorded along the entire tank at different times.

To simplify the discussion of the results, the obtained wavecuts are split into different phases

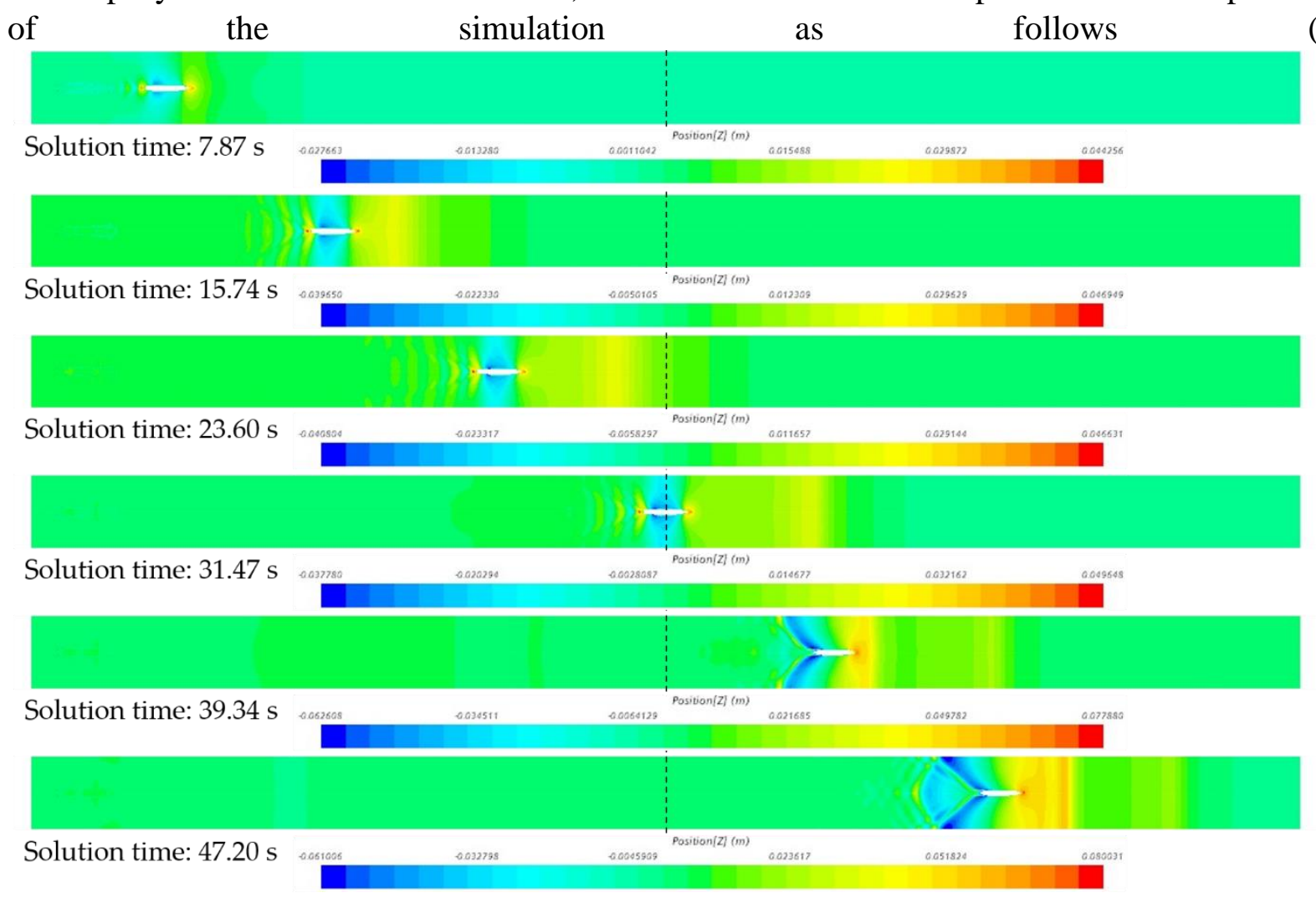

Figure 


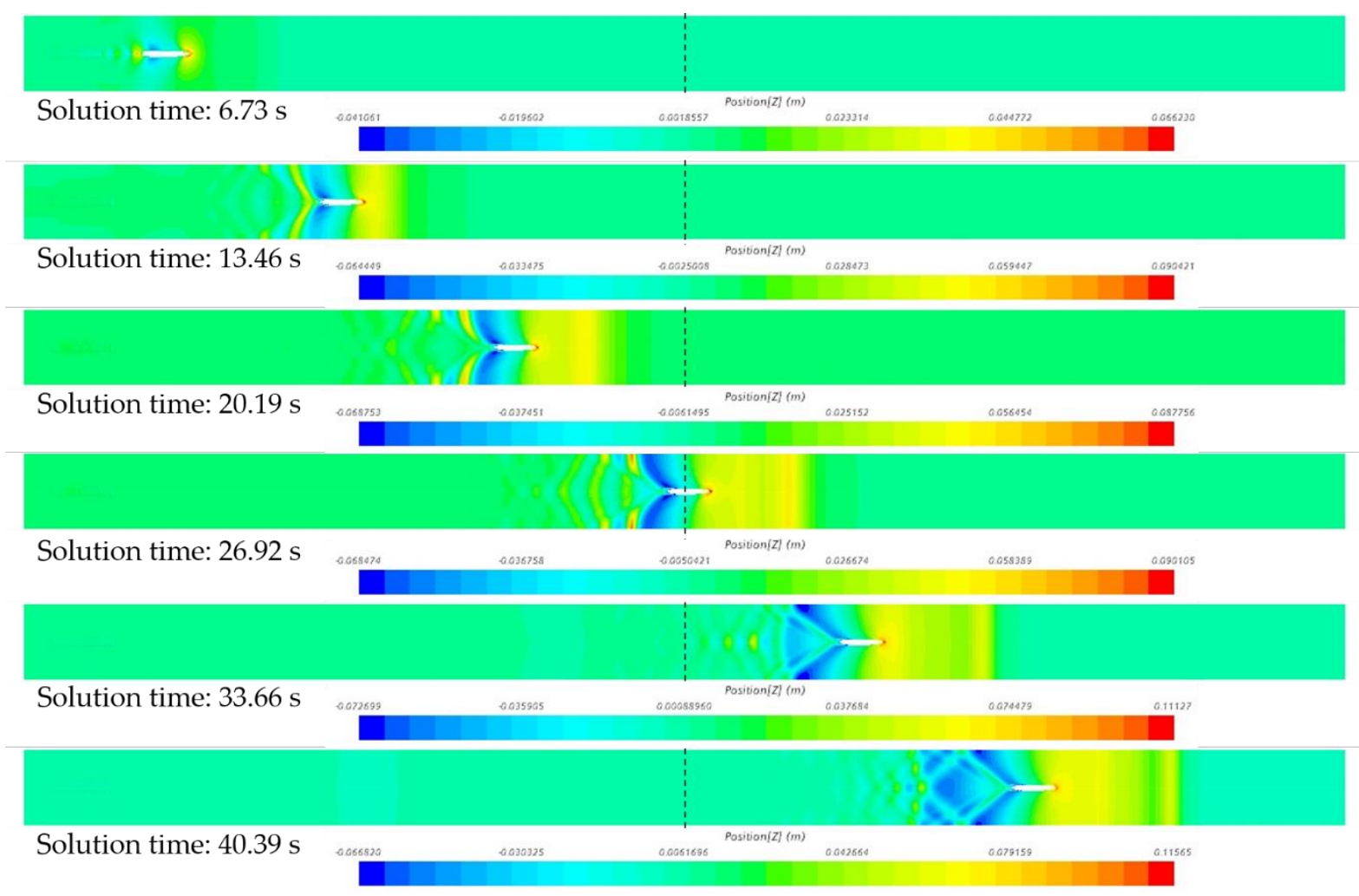

Figure 6 can also be consulted in this respect):

I. End of acceleration - this occurs when the ship has reached its target speed. The wavefield at this stage is not yet fully developed and differs from its pseudo-steady state in several important ways. Discussion of these can be found in Doctors (1975) and Day et al. (2009).

II. Subcritical wavefield development - this phase of the simulation contains the time required by the wavefield to approach its steady state. This process occurs in all towing tanks, whether virtual or physical. In numerical tanks where the ship's position in the $x$ direction is maintained constant, this phase is equivalent to the time allowed for convergence.

III. Prior to the step - at this stage, the ship begins to interact with the step. Initially, this is indirectly via the bow wave, which is partially compressed by the additional blockage.

IV. Transiting the step - this phase occurs while the step is located under the ship itself.

V. After the step - this phase begins as soon as the stern of the ship has cleared the step. Interactions between the depth transition and the step do not cease here. Instead, the accelerated fluid aft of the ship, interacts with the step continuously for a considerable time. This effect is subsequently demonstrated.

VI. Critical wavefield development - once the ship has cleared the step and advanced about one ship length along the canal, the wavefield corresponding to the depth $h_{s}$ has begun developing. This can be thought of in similar terms as explained in II, i.e. a convergence stage.

The development of the wavefield is split into the above stages and given in Figure 9 and Figure 10 for case $1\left(F_{h i}=0.77, F_{h s}=1\right)$ along $y / \mathrm{w}=0.1$ and $y / \mathrm{w}=0.2$. In these figures, the maximum and minimum wave elevation for each phase is recorded and marked. Evidently, for $y / \mathrm{w}=0.2$, shown in Figure 10, the disturbance caused by the ship decays in the $y$ direction, allowing the 
soliton to assume the maximum value in phases II, III, and IV. For $y / w=0.1$ (Figure 9), this is only the case in phase II.

An interesting property, observed in Figure 9 and Figure 10 relate to the difference of the wave field aft of the ship in phases III and IV (prior and in transit of the step) when compared to phase $\mathrm{V}$ (after the step). The oscillatory pattern observed in the earlier phases, corresponding

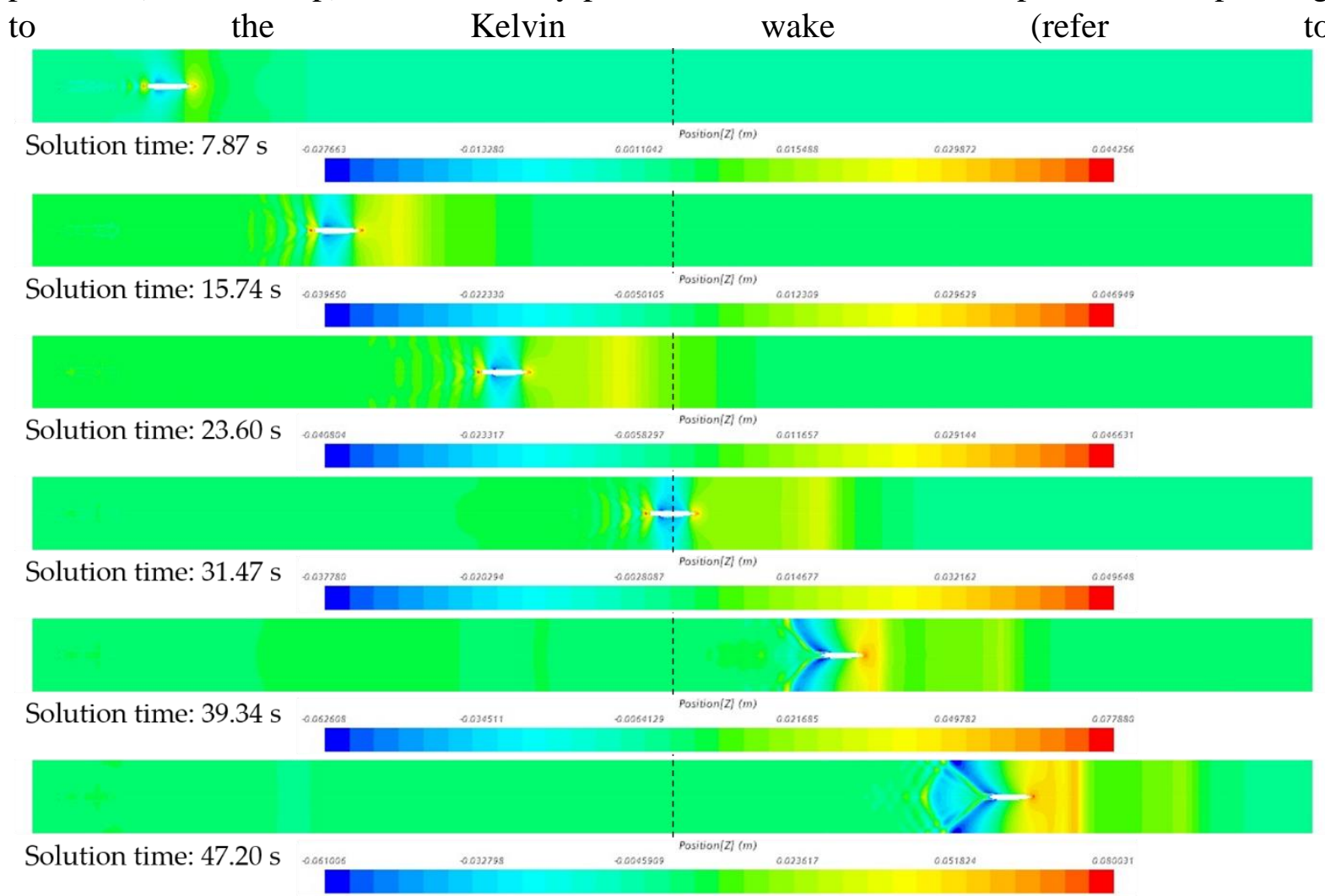

Figure 5 for a top view) are transformed as the ship enters the region of depth $h_{s}$. These are replaced by a substantial depression, following the ship, as evident in the final stage given in Figure 9 and Figure 10 along each wavecut. The length and height of the wave, trapped at the ship's stern is seen to decrease substantially, while the bow wave is considerably stronger in both respects. This observation partly explains the increase in resistance, shown in Figure 4. Namely, the ship carries with it a greater volume of water at its bow. 

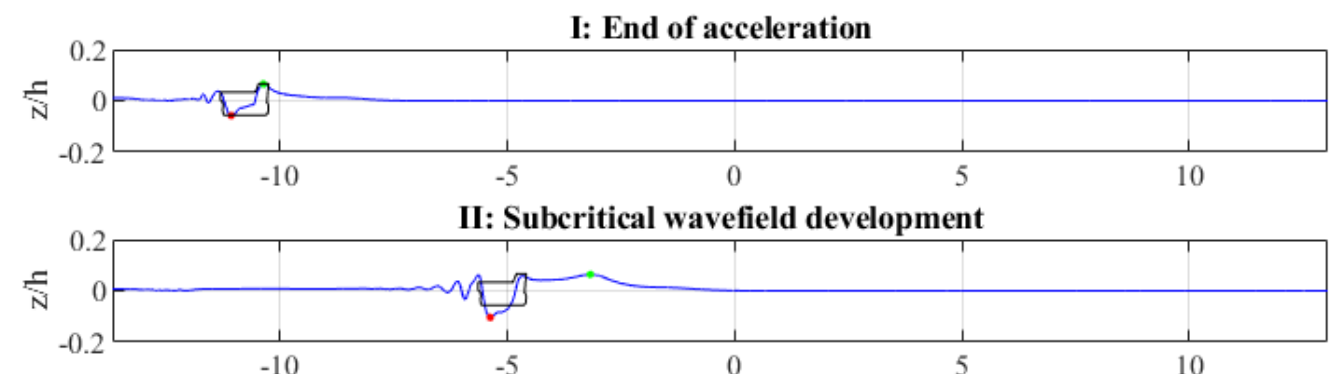

III: Prior to the step
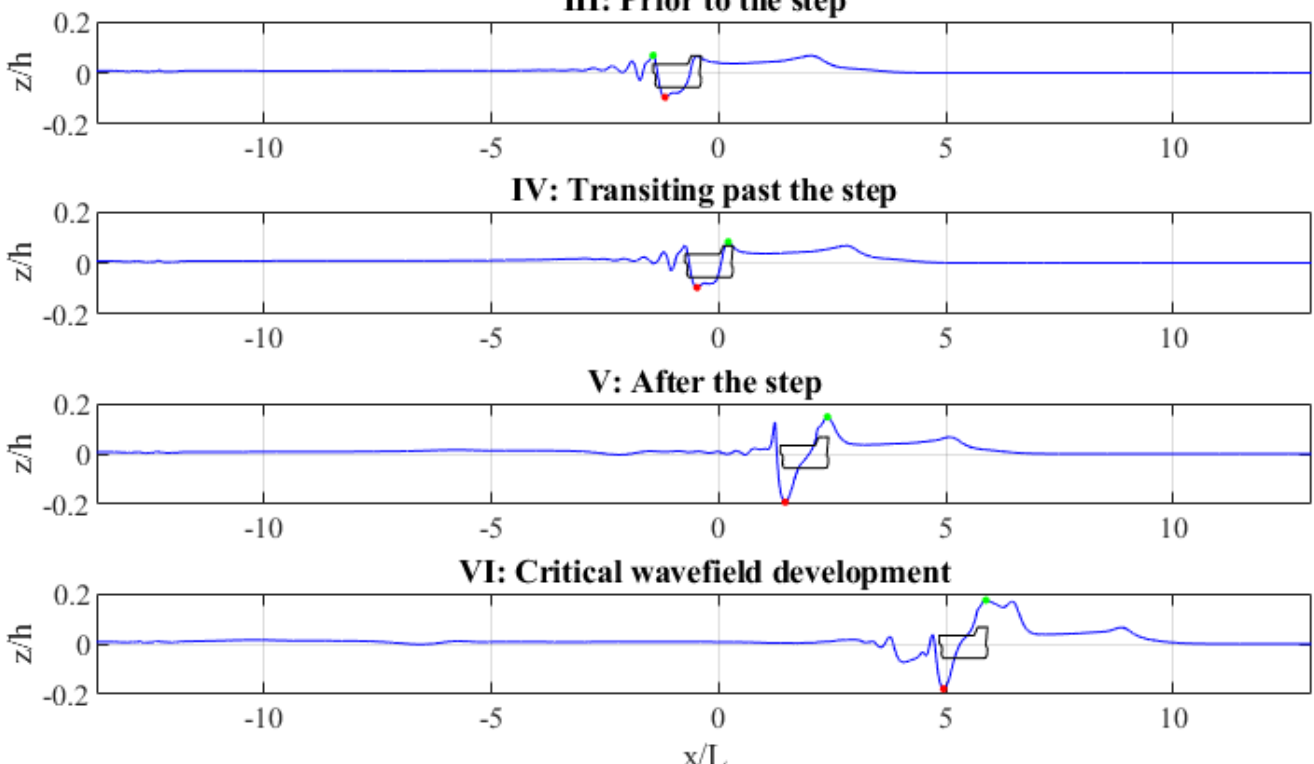

Figure 9. Wavecut $1(y / w=0.1)$ evolution for case $F_{h i}=0.77, F_{h s}=1$, made dimensionless by the initial depth $h_{i}=0.32$. Maxima and minima are marked with green and red points, respectively. Note that the ship outline has been scaled down by a factor of 8 in the vertical direction to enable a visualisation of the ship's position relative to the wavecut.

In investigating the wavefield, it is important to keep in mind that the RANS solver models the flow in a fully nonlinear manner. In a recent piece of work, the authors demonstrated that the present set-up models the dispersive properties of the Kelvin wake in good agreement with the linear dispersion relation (Terziev et al., 2020). In the present work, the opportunity to compare the evolution of the numerical wavefield with linear potential flow approximations of wave transmission past different step is used to provide a form of validation.

As stated earlier, Lamb (1932), Bartholomeusz (1958), and Newman (1965) all arrived at the same relationship describing the transmission coefficient, expressed as shown in Eq. (6):

$T_{R}=\frac{2 \sqrt{h_{i}}}{\sqrt{h_{i}}+\sqrt{h_{s}}}$

where $T_{R}$ is ratio of transmitted and incident wave height. This relationship follows directly from the wave speed in each shallow water region, whose linear form is $\sqrt{g h}$, with $g$ being the gravitational acceleration. Lamb (1932) showed that this relationship can be arrived at simply by imposing continuity and equivalence of the two waves (transmitted and reflected component) at the point directly above the step. All possible values of $T_{R}$ are shown in Figure 
11 alongside the numerical predictions for cases 1-4 using this method. Here, the theoretical predictions are marked along each line representing the possible coefficient values to enable a better visualisation of the numerical results and their deviation from the theory.
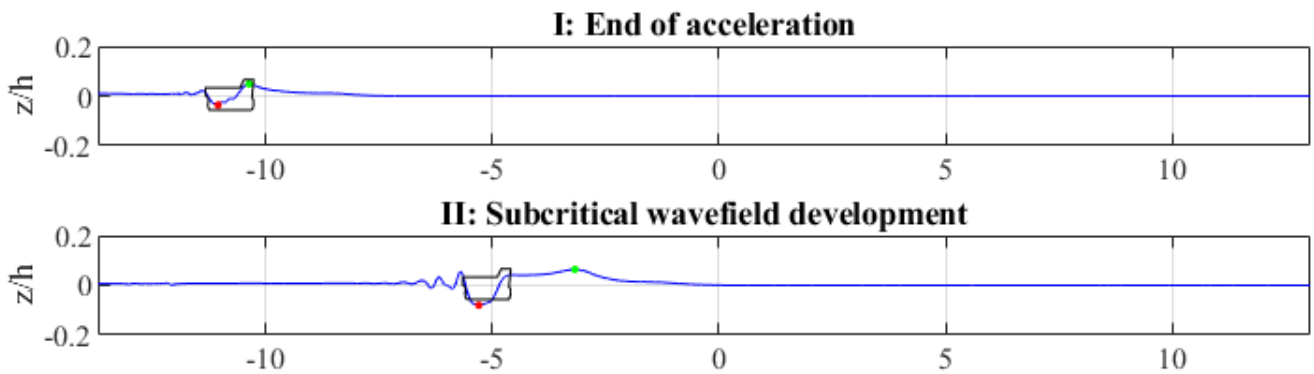

III: Prior to the step

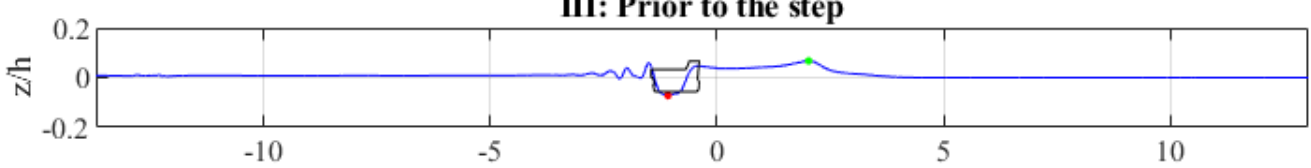

IV: Transiting past the step
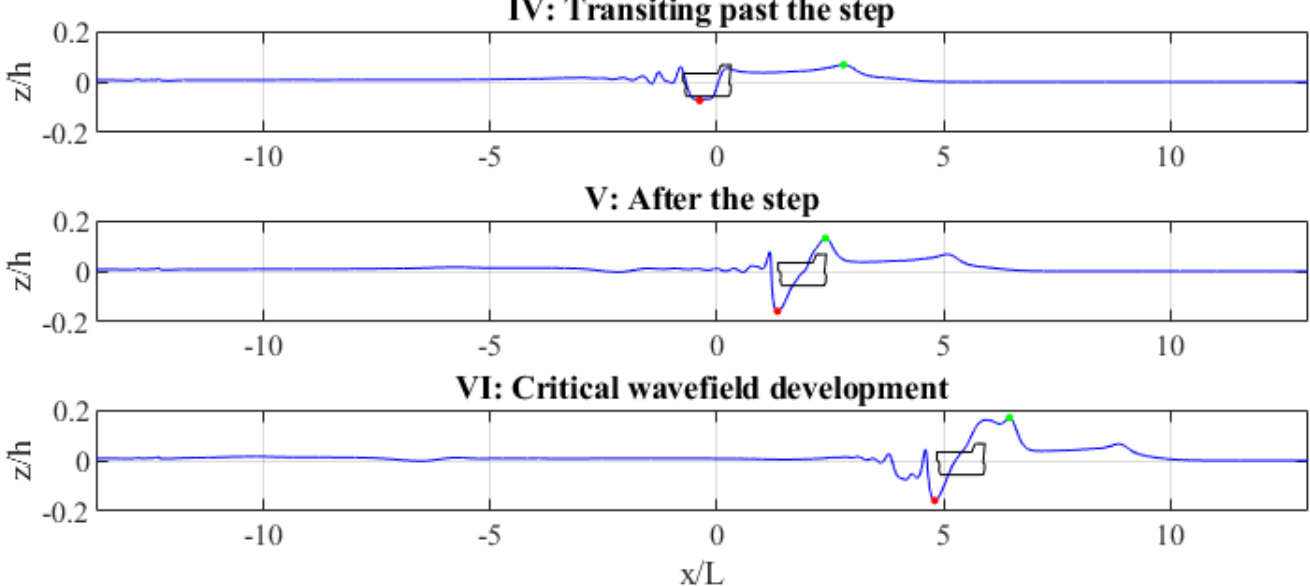

Figure 10. Wavecut $2(y / w=0.2)$ evolution for case $F_{h i}=0.77, F_{h s}=1$, made dimensionless by the initial depth $h_{i}=0.32$. Maxima and minima are marked with green and red points, respectively. Note that the ship outline has been scaled down by a factor of 8 in the vertical direction to enable a visualisation of the ship's position relative to the wavecut. 


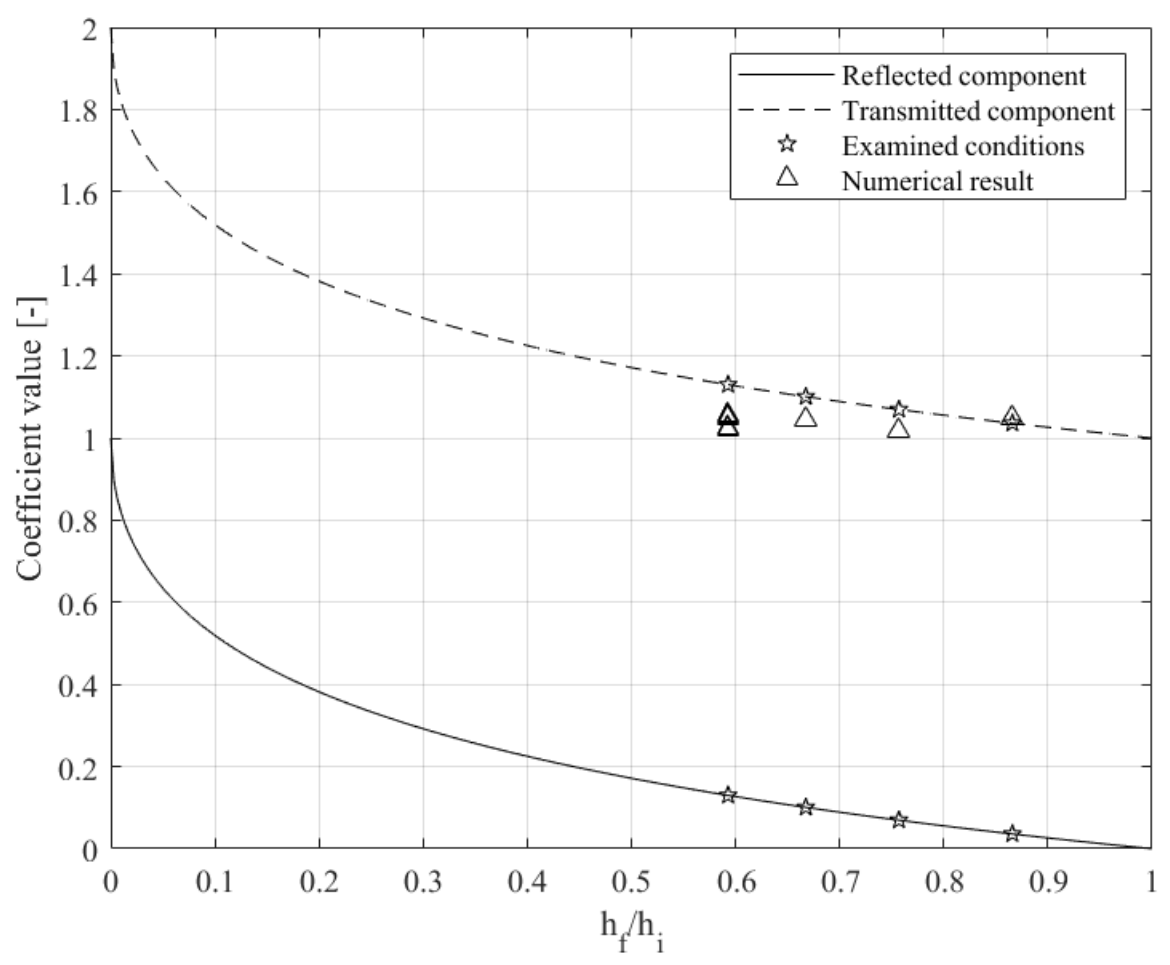

Figure 11. Transition and reflection coefficients for cases $1-4\left(F_{h i}=0.77\right)$.

Figure 11 also contains the reflected coefficient values for the entire range depth ratios. In this case, no numerical predictions are given because a reflected wave elevation is not observed. The suspected cause of this is the ship's interaction with the wavefield. To elaborate, any reflected wave will be disturbed almost as soon as it is created by the passing of the ship. On the other hand, the numerical results for $T_{R}$ show good agreement with the theoretical line. In fact, the present datapoints are less scattered than the experimental results shown in Newman (1965). It should be noted that in the aforementioned work, the author used the infinitely deep initial region theory to construct his line. Nevertheless, he demonstrated that the experimental data is scatted around the line, providing a form of validation for the present wavefield.

The reason why several closely positioned datapoints are shown in Figure 11 relates to the manner in which the wavefield is sampled. Specifically, Figure 9 and Figure 10 show that one has a range of choices when it comes to taking the incident wave height and transmitted wave height. Therefore, it is thought important to demonstrate that this particular choice is of little importance on the positioning of the calculated transmission coefficients. For this reason, only one datapoint is given for cases II - IV. To further investigate the significance of the location over which the soliton is taken, the wavefield maximum and minimum along the wavecuts given in Figure 9 and Figure 10 are shown in Figure 12. 

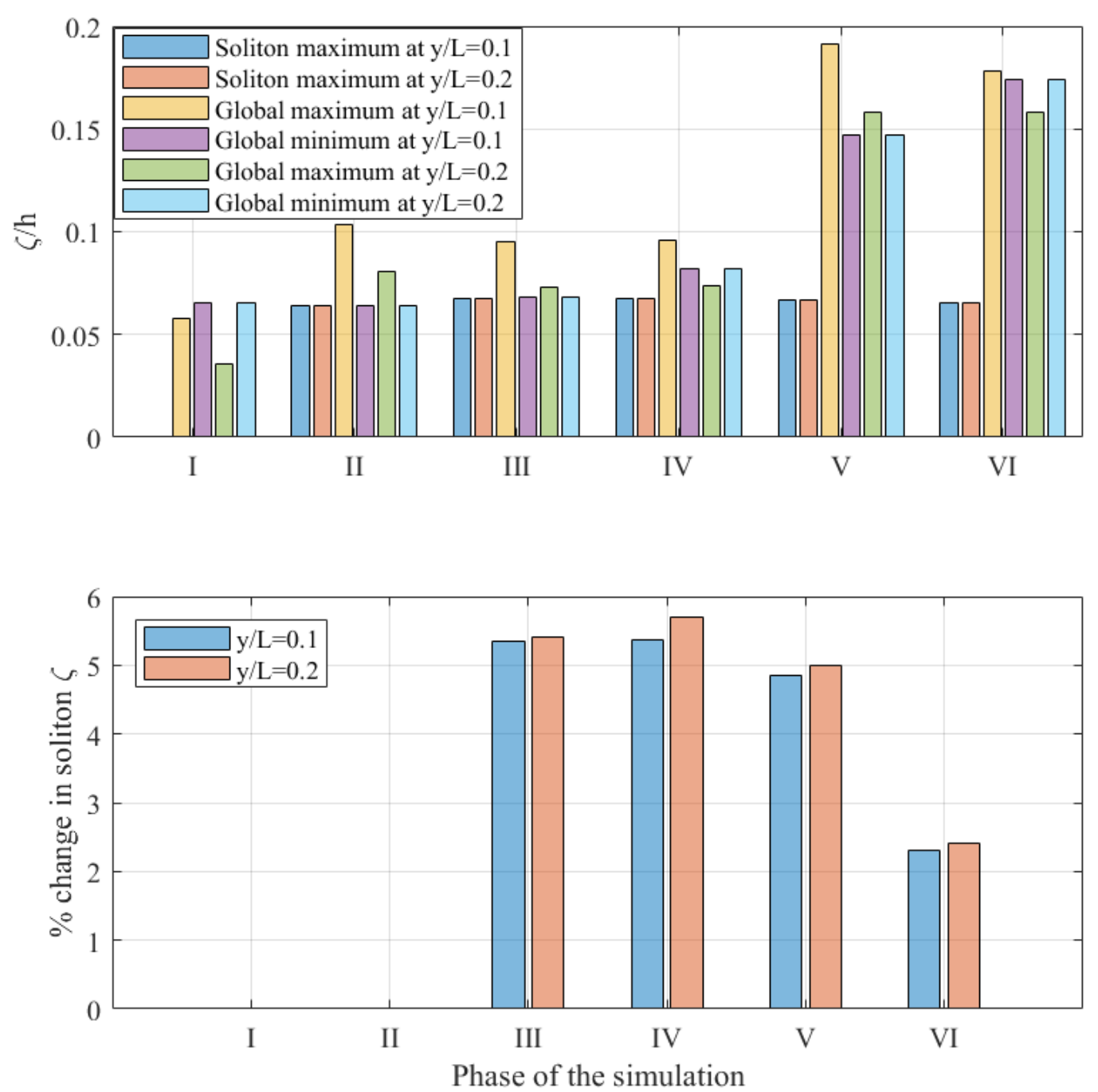

Figure 12. Analysis of the wavefield for $F_{h i}=0.77, F_{h s}=1$ along wavecuts $y / \mathrm{w}=0.1$ and $y / \mathrm{w}=0.2$

For the purposes of this work, a simple relation was used to predict the transmitted component of the soliton, which was shown to agree well with the numerical results. However, it also important to state that following the work of (Newman, 1965), (Lamb, 1932), and (Bartholomeusz, 1958), considerable developments have been made in the field of predicting the behaviour of waves at depth transitions. Some recent studies include (Bender and Dean, 2003), and discussions thereof (Bender and Dean, 2005; Liu and Lin, 2005), which can give the reader a much more in-depth appreciation of the complexities encountered in the related field. Many methods, such as that of Marshall and Naghdi (1990) use the wavenumber, $k$, in each region to arrive at an expression for $T_{R}$.

To estimate the wavenumber, Lee and Lee (2019) give several methods, also see Guo (2002), and Newman (1990). In the present work, the approach of Hunt (1979) and Havelock (1908) are used. These techniques agree well for the examined range $\left(F_{h i}=0.77\right)$, as shown in Figure 13. 


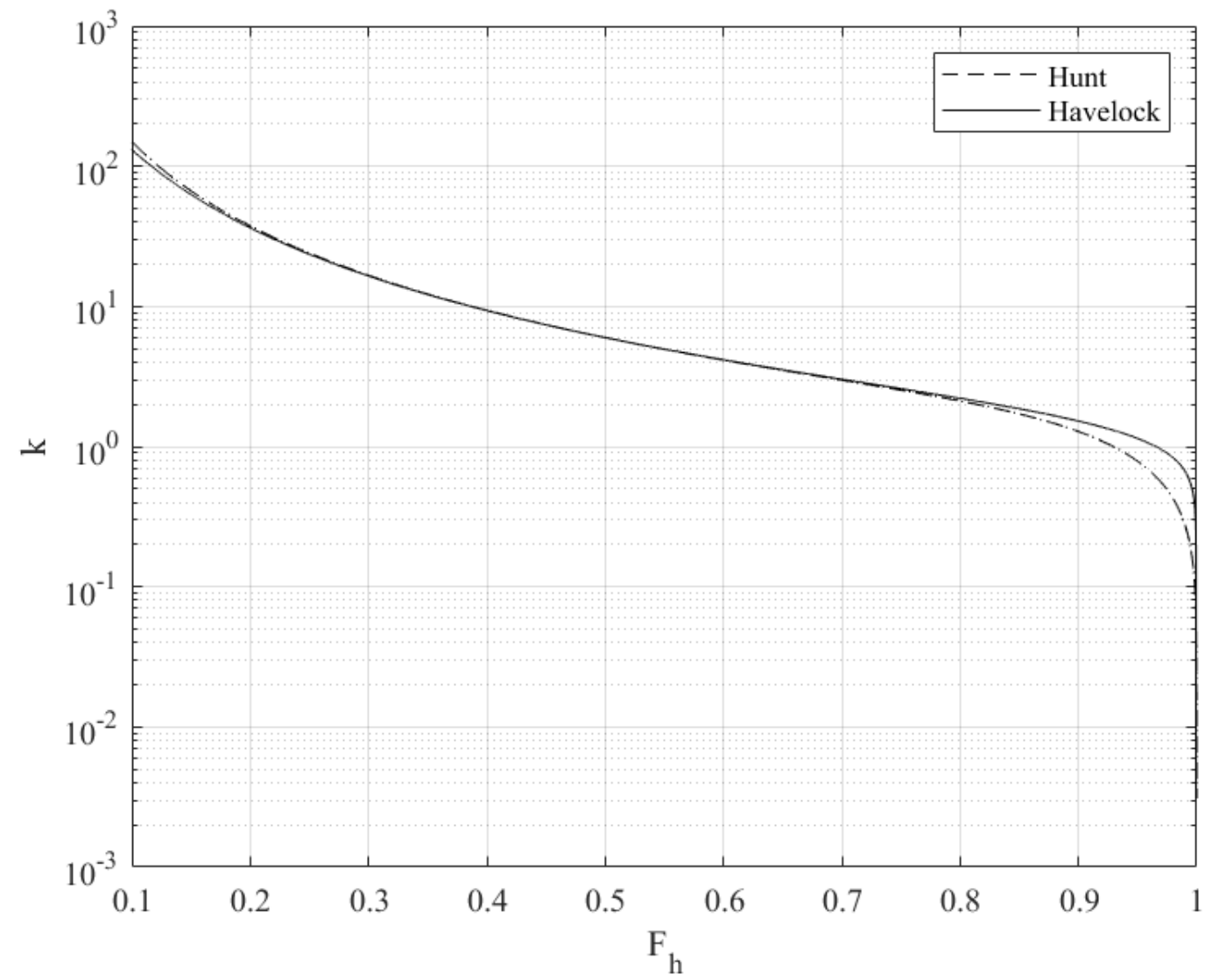

Figure 13. Predicted wavenumber $\left(k_{i}\right)$ for each depth Froude number

Clearly, one runs into problems using these methods when predicting the wavenumber in the region past the step if $F_{h s}=1$. In this respect, Marshall and Naghdi (1990) proposed the following relationships between the two regions' wavenumbers:

$k_{i} \tanh \left(k_{i} h_{i}\right)=k_{s 1} \tanh \left(k_{s 1} h_{s}\right)$

$k_{s 2}=\sqrt{\frac{h_{i} k_{i}^{2}}{h_{s}\left(1+k_{i}^{2} h_{i}^{2} / 3-k_{i}^{2} h_{i} h_{s} / 3\right)}}$

$k_{s 3}=k_{i} \sqrt{h_{i} / h_{s}}$

Then, the reflected transmitted and coefficients are given in Eq. (10) and Eq. (11), respectively:

$R_{R}=\frac{k_{s}-k_{i}}{k_{2}+k_{i}}$

$T_{R}=\frac{2 k_{s}}{k_{S}+k_{i}}$

In Eq. (7) - Eq. (9), the subscripts 1, 2, 3 are used to differentiate the wavenumber predictions. To examine the predictions graphically, Figure 14 was constructed to show the relationships between $k_{s 1-3}$ and the wavenumber, as predicted by Hunt's (1979) method. 


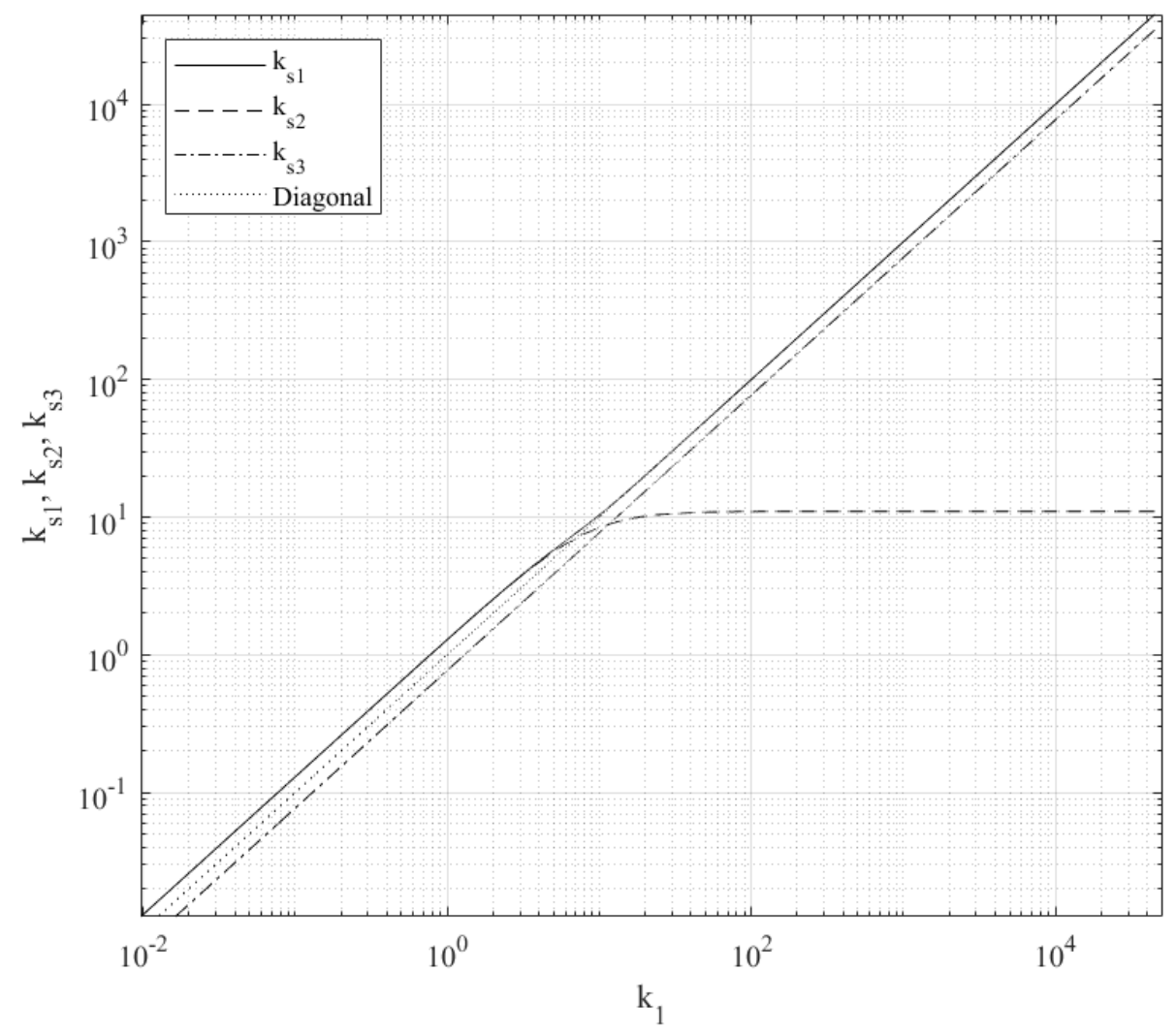

Figure 14. Wavenumber predictions for the region past the step. Depicted: case $1, F_{h i}=0.77$ and $F_{h s}=1$.

As $k_{s 2}$ increases past a value of 100 , it essentially ceases to grow. Fortunately, the values of interest in the present study are far from this boundary. The predicted wave numbers using the aforementioned methods are summarised in Table 5.

Table 5. Summary of wavenumbers for cases $1 \sim 4\left(F_{h i}=0.77\right)$.

\begin{tabular}{|c|c|c|c|}
\hline Method & \multicolumn{2}{|c|}{ Description } & Wavenumber value \\
\hline Havelock (1908) & \multirow{15}{*}{$F_{h i}=0.77$} & \multirow{3}{*}{ Initial region } & 7.591 \\
\hline Hunt (1979) & & & 7.367 \\
\hline Relative difference & & & $-3.04 \%$ \\
\hline \multirow{4}{*}{ Eq. (7) } & & $F_{h s}=1$ & 7.974 \\
\hline & & $F_{h s}=0.943$ & 7.777 \\
\hline & & $F_{h s}=0.885$ & 7.608 \\
\hline & & $F_{h s}=0.826$ & 7.470 \\
\hline \multirow{4}{*}{ Eq. (8) } & & $F_{h s}=1$ & 7.224 \\
\hline & & $F_{h s}=0.943$ & 7.093 \\
\hline & & $F_{h s}=0.885$ & 7.031 \\
\hline & & $F_{h s}=0.826$ & 7.085 \\
\hline \multirow{4}{*}{ Eq. (9) } & & $F_{h s}=1$ & 5.672 \\
\hline & & $F_{h s}=0.943$ & 6.018 \\
\hline & & $F_{h s}=0.885$ & 6.409 \\
\hline & & $F_{h s}=0.826$ & 6.855 \\
\hline
\end{tabular}


Since Marshall and Naghdi (1990) derived Eq. (7) without additional assumptions, it is used to construct transmission coefficients shown in Figure 15 for cases $1 \sim 4\left(F_{h i}=0.77\right)$. Here, the range has been retained from Figure 11 for consistency. Figure 15 demonstrates that the much simpler approach of given in Figure 11 can be used to quickly estimate with essentially the same accuracy the transmission coefficients. This is valid due to the particular case studies selected. Had a deeper water region been chosen, the approach of Marshall and Naghdi (1990) is recommended.

At this stage it is also worthwhile to mention that the decrease in height of the soliton with distance, given in Figure 12 is also physically sound. As stated earlier, the height of the wave in front of the ship was shown to decay. This was given as a justification as to why there are several datapoints for $F_{h s}=1$ in terms of transmission coefficients. The dissipation of the wave is due to a combination of viscous action in the fluid and friction at the edges and bottom of the tank. Here, the reader is reminded that all boundary conditions, with the exception of the symmetry plane and overset box are no-slip walls. Therefore, the dissipation of the wave is an expected outcome.

Dissipation in the present context can be approximated as shown in Figure 16. Here, Eq. (12) and Eq. (13) are used to construct the dissipation and amplitude change parts of the plot, as reported in Lamb (1932). Further discussion of these equations can be found in the relevant literature (Denner et al., 2017; Dorn, 1966; Hunt, 1964; Keulegan, 1948; Liang and Chen, 2019)

$D=\frac{2 k}{b} \sqrt{\frac{v}{2 \omega}} \frac{k w+\sinh (2 k h)}{2 k h+\sinh (2 k h)}$

$\delta=\zeta e^{-D \times x}$

where $D$ is the dissipation, $\omega^{2}=g k \tanh (k h), v$ is the kinematic viscosity, $\delta$ is the amplitude of the wave having travelled $x$ metres, and $\zeta$ is the elevation of the initial wave. It should be noted that $k_{s 1}$, as given in Table 5 and Eq. (7) are used throughout for consistency. 

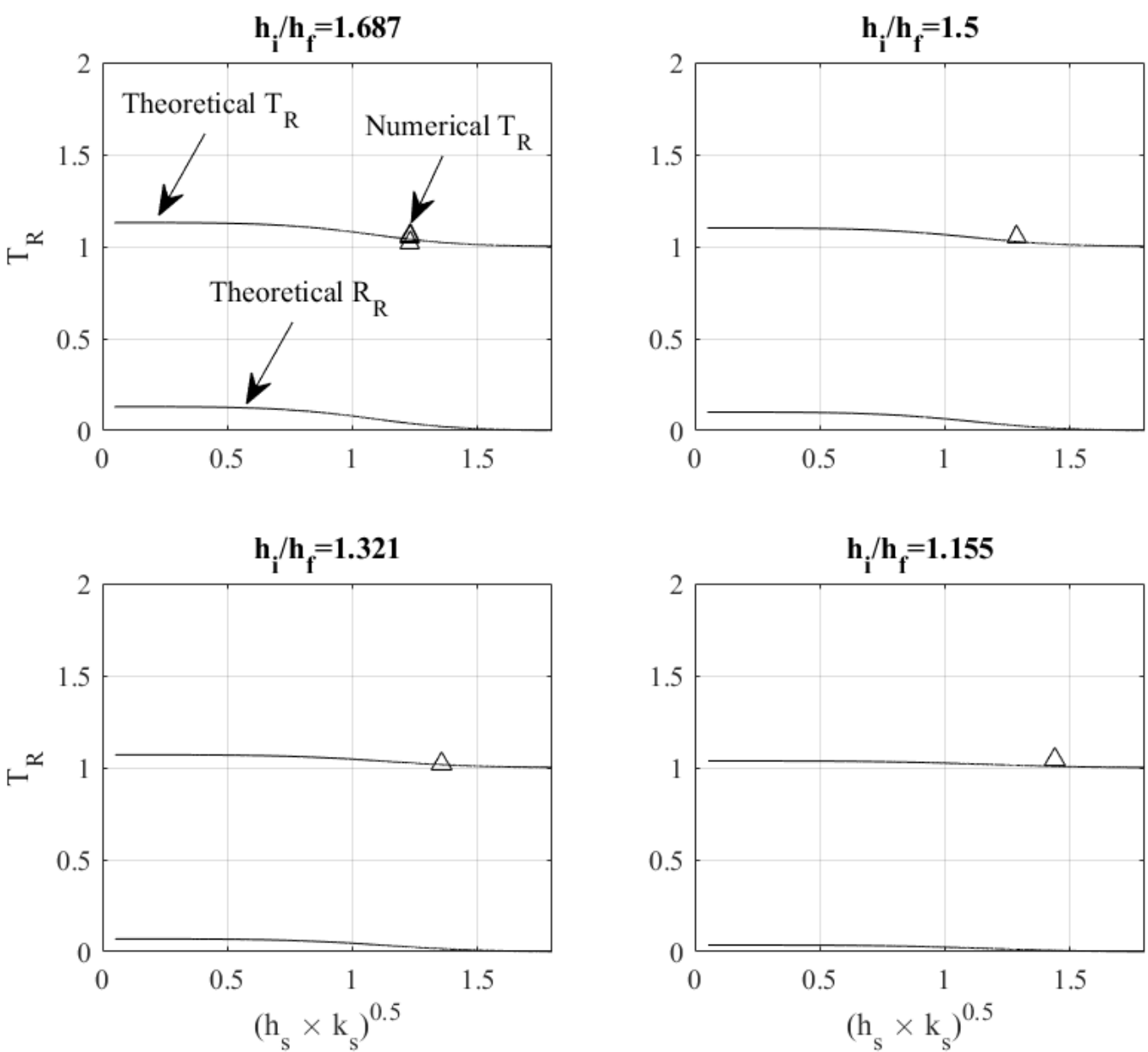

Figure 15. Transmission and reflection coefficients based on Marshall and Naghdi's (1990) $\operatorname{method}\left(F_{h i}=0.77\right)$.

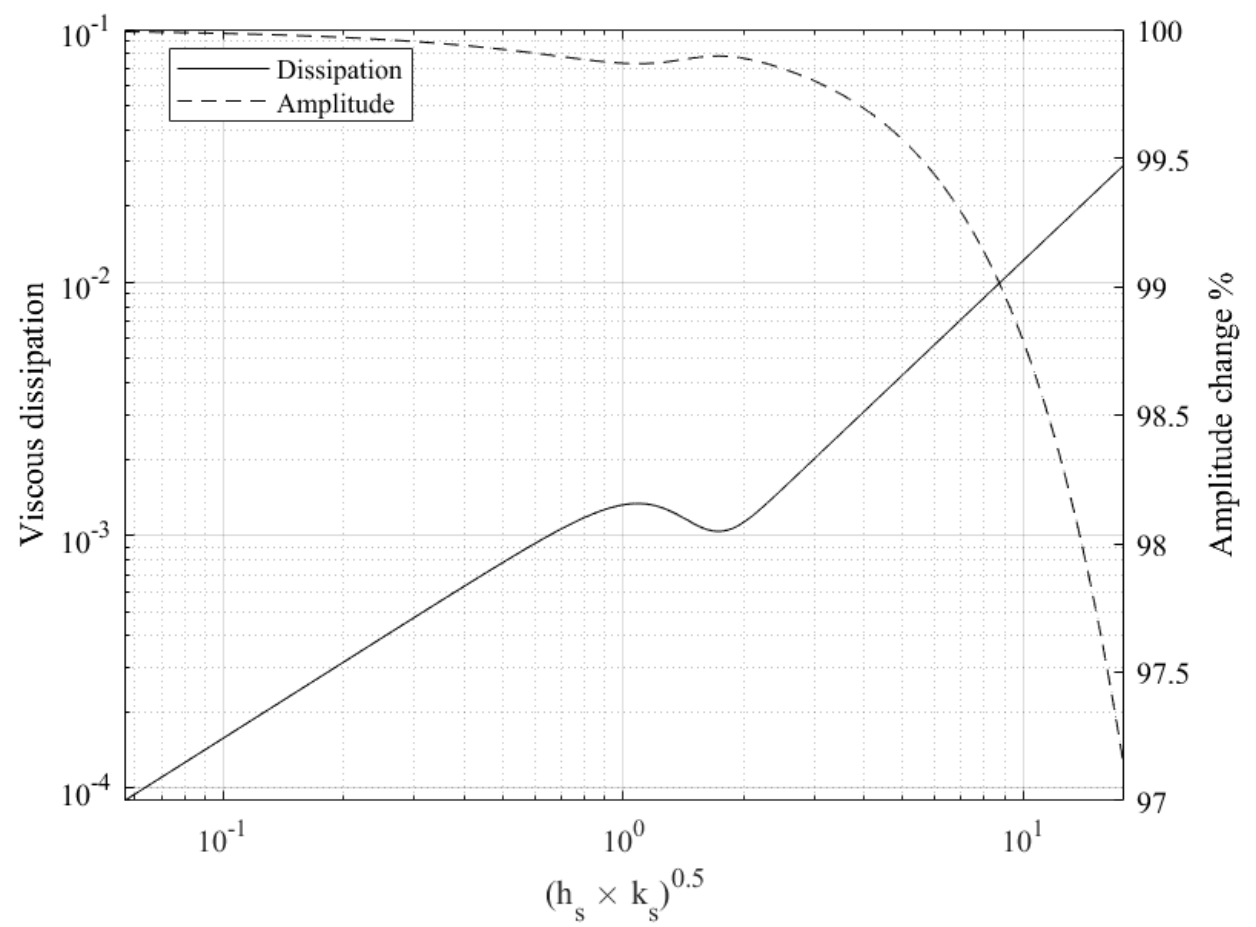


Figure 16. Viscous dissipation on a unit wave with different dispersive properties travelling a unit distance.

In this work, it is not immediately obvious where the origins of the soliton lie. It is therefore difficult to determine an exact damped amplitude. Thus, tracing the soliton's decay continuously and comparing it with the analytical solution is not attempted. Instead, we use the value of the soliton after it has transferred onto the step. Then, the damping relations given in Eq. (12) and Eq. (13) are applied to arrive at a decrease in magnitude by $2.923 \%$. By contrast, the numerical result is that the soliton reduced in magnitude by $2.994 \%$ between phases III (prior to the step) to VI (critical wavefield development). Note that the soliton has already cleared the step at phase III. This agreement is excellent and indicates that the present solution has captured the physics of wave propagation and transmission well, as indicated by the results given here and in Figure 11 and Figure 15.

In this work, the numerical modelling of the wavefield, including the friction on the side walls and bottom is shown to agree well with analytical solutions. Therefore, the use of wall functions at the boundaries of the tank is shown to provide sufficient accuracy. This result also suggests that numerical diffusion, incurred by the grid density is minimal for the soliton.

Another aspect of the generated data considered here relates to the velocity field produced by the soliton, the ship and its wave system, as they interact with the step. As was the case earlier, we focus on the critical transition case (case $1, F_{h i}=0.77$ and $F_{h s}=1$ ). To examine the velocity field, the proportion of the domain beneath the undisturbed free surface is plotted at various times in Figure 17. The symmetry plane is used as a reference in this case throughout. Longitudinally, one ship length before and after the step is included in the plots.
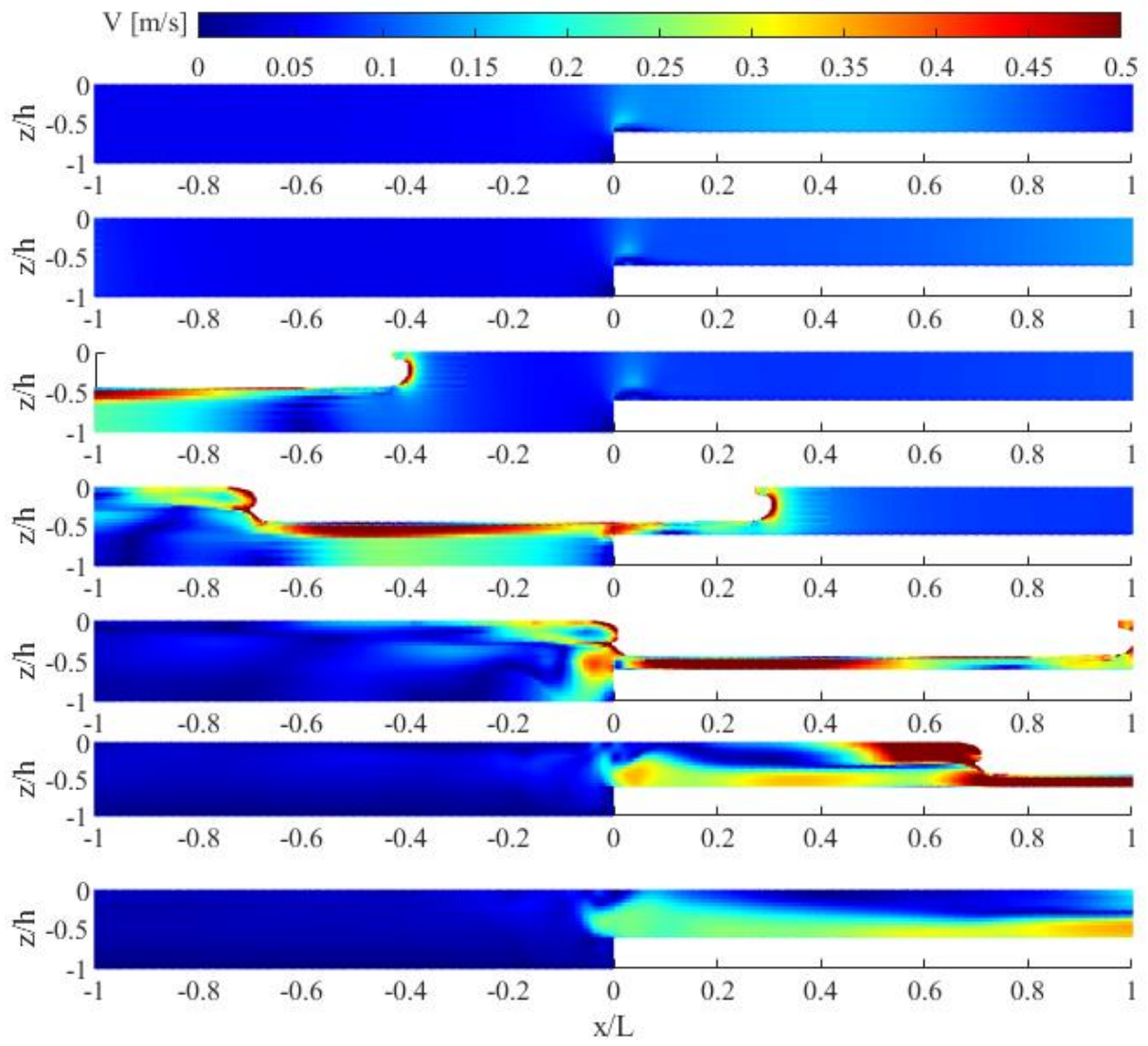
Figure 17. Generated velocity field for case $1, F_{h i}=0.77$ and $F_{h s}=1$ as the ship and soliton interact with the step.

The first part of Figure 17 shows the process of the soliton as it has cleared the step. Here, the step causes a region of elevated velocity magnitude to persist even after the soliton has propagated a full ship length past the depth reduction. This would likely persist for a long time had the ship not disturbed the flow field. The most surprising aspect of the solution is that a significant proportion of the fluid maintains its velocity a significant time after the ship has cleared the step. The flow field in the final part of Figure 18 is in the direction of the ship, with a vortex persisting at the step's edge.

The observations made in Figure 18 are in agreement with recent numerical and experimental work, which demonstrated that a boundary layer will form on the seabed in very shallow conditions (Böttner et al., 2020; Shevchuk et al., 2016). The present work also demonstrates this effect in the final part of Figure 17. However, as mentioned earlier, the domain does not feature any inlets nor outlets. Therefore, any fluid, accelerated in the ship's direction must return to equalise the pressure and water elevation behind the ship. Ship-generated waves would also contribute to this, but they diffuse by a combination of sides/bottom friction, numerical and viscous dissipation. Indeed, the smaller the waves, the greater the action of numerical dissipation.

To investigate the mechanism by which the fluid returns after being accelerated in the direction of the ship, the velocity field at the step is taken at the end of the simulation (i.e. once the ship's bow is about $1 \mathrm{~m}$ from the end boundary) and shown in Figure 18. Although the magnitude of velocity is given in the contour of the plot, the $x$-direction velocity is also shown as a vector field. This reveals that fluid is returning at a palpable rate to the step. However, once the step is cleared, the velocity diminishes rapidly. Such an effect may explain the elevated time-history of the free surface in Figure 7 and Figure 8. Specifically, these indicate that considerable volumes of water have been swept along with the ship and are subsequently returning to equilibrium.

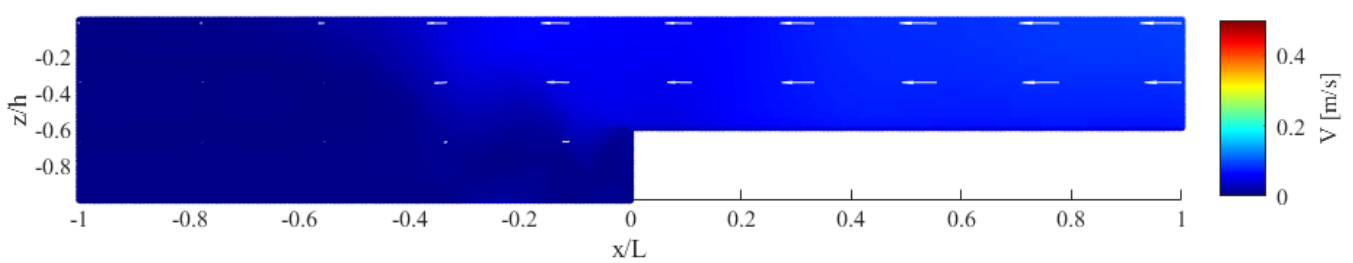

Figure 18. Example velocity field near the step at the end of the simulation for case 1:

$$
F_{h i}=0.77, F_{h s}=1 \text {. }
$$

A final consideration is given to the wavecuts shown in Figure 9 and Figure 10. Although difficult to spot, in phase VI (critical wavefield development), a wave trough can be seen propagating in the direction, opposite the ship's. This is not a purely numerical phenomenon. Grue's (2017) theory and observations in a Norwegian fjord demonstrated this is a key feature of depth transitions. In the case depicted in Figure 9 and Figure 10, the depression is of approximately $-40 \mathrm{~cm}$ height when converted to full-scale. This consequence of depth transitions is clearly an important part of the physics and has the potential to cause severe infrastructure damage.

\section{Conclusion and recommendation for future research}


This study examined the impact of a step change in the water depth on ship performance using the commercial RANS solver Star-CCM+. To the best of the authors' knowledge, this work, using CFD to study depth transitions over a ship's track, is the first of its type in the field. The adopted case studies reflected a narrow canal, along the length of which, the water depth changes abruptly. This scenario was modelled to reflect recent work performed experimentally (Elsherbiny et al., 2019b) over a constant canal cross-section, and subsequently validated by the authors (Terziev et al., 2020). Four depth reductions were modelled with two constant speeds. These were deliberately selected to provide transcritical depth Froude numbers, with one case targeted specifically at the critical speed.

The results were reported in terms of percentage increase of the base resistance encountered by the ship prior to and after the step. These indicated that a resistance increase of up to approximately $226 \%$ may occur if the transition results in subcritical depth Froude numbers approaching the critical speed. On the other hand, it was shown that when the ship has a high initial speed, close to the sub-supercritical boundary, the resistance increase is considerably milder. Results indicated in this case a change of less than $50 \%$ for the model scale ship.

In cases where the initial speed is near $F_{h}=1$, it was shown that the wavefield is largely twodimensional and unaffected by the height of the step. On the other hand, when the speed is lower, the interaction of the ship's wavefield with the depth transition is highly sensitive to the step height. For this reason, the transition of waves past shallow water depth discontinuities were examined in some detail. Two theories with varying complexity were employed, both of which agreed well with the numerical results. This suggested that the shallow water celerity approximation $\sqrt{g h}$ holds well even when the critical depth Froude number is reached in water depths up to $h / T=2.2$.

There are several aspects of the present work the authors would extend. Firstly, the geometry of the depth transition is expected to be of high significance to the results. The same study would also be interesting if the width were decreased either independently of or along with the depth. Transitions into deeper water are of equal interest, since the formed boundary layer of the bottom in the shallow region would spill over into deeper water.

Simultaneously, the damping of the soliton was shown to be in excellent agreement with analytical relations for viscous dissipation of waves in canals. The theoretical result for damping in the amplitude of the soliton was $2.923 \%$, whereas the numerically obtained decrease in amplitude was $2.994 \%$. This suggests that the present RANS approach can model the dissipation of solitons with high accuracy. It was also shown that a boundary layer is formed on the canal bottom, which persists long after the ship has passed through. The accelerated fluid also requires considerable time to return to its quiescent state.

In terms of numerical uncertainty, it was observed that the requirements for the grid are considerably higher. The numerical uncertainty analysis was performed for both regions, before and after the step. This revealed that as the depth Froude number decreased, the requirements in terms of mesh increased noticeably, resulting in an elevated uncertainty. On the other hand, both regions showed relatively low temporal dependency.

Strategies to minimise or eliminate the generated soliton can be numerically explored. Eliminating the soliton altogether would have applications in towing tanks. The manner in which the ship is accelerated is thought to be the primary cause of this. Different profiles of the 
velocity curve should be investigated, as well as the relative influence of turbulence modelling on ship performance in this type of towing tank. Finally, a study on the effect of $y^{+}$treatments of the background walls would be of interest. Although it is not thought that this particular metric impacts the soliton, it may be of consequence to the ship and the formation of boundary layers on the bottom.

\section{Acknowledgements}

Results were obtained using the ARCHIE-WeSt High Performance Computer (www.archiewest.ac.uk) based at the University of Strathclyde. The work reported in this paper is drawn from the first author's PhD thesis. The first author gratefully acknowledges the scholarship provided by the Faculty of Engineering at the University of Strathclyde, which fully supports his $\mathrm{PhD}$. 


\section{References}

Alam, M.-R., Mei, C.C., 2008. Ships advancing near the critical speed in a shallow channel with a randomly uneven bed. J. Fluid Mech. 616, 397-417. https://doi.org/10.1017/S0022112008004035

ASME (American Society of Mechanical Engineers), 2009. Standard for Verification and Validation in Computational Fluid Dynamics and Heat Transfer - ASME V\&V 20-2009, ASME International.

Bartholomeusz, B.Y.E.F., 1958. Reflexion of long waves at a step. Math. Proc. Cambridge Philos. Soc. 54, 106-118.

Bechthold, J., Kastens, M., 2020. Robustness and quality of squat predictions in extreme shallow water conditions based on RANS-calculations. Ocean Eng. 197, 106780. https://doi.org/10.1016/j.oceaneng.2019.106780

Beji, S., 2018. Kadomtsev-Petviashvili type equation for entire range of relative water depths. https://doi.org/10.1080/21664250.2018.1436241org/10.1080/21664250.2018.1436241

Bender, C.J., Dean, R.G., 2005. Reply to discussion of "Wave transformation by twodimensional bathymetric anomalies with sloped transitions" [Coast. Eng. 50 (2003) 61 84]. Coast. Eng. 52, 201. https://doi.org/10.1016/j.coastaleng.2004.11.003

Bender, C.J., Dean, R.G., 2003. Wave transformation by two-dimensional bathymetric anomalies with sloped transitions. Coast. Eng. 50, 61-84. https://doi.org/10.1016/j.coastaleng.2003.08.002

Benham, G., Benzaquen, M., Clanet, C., 2020. arXiv : submit / 3068148 [ physics . flu-dyn ] 1 Mar 2020.

Benham, G.P., Boucher, J.P., Labbé, R., Benzaquen, M., Clanet, C., 2019. Wave drag on asymmetric bodies. J. Fluid Mech. 878, 147-168. https://doi.org/10.1017/jfm.2019.638

Böttner, C.U., Anschau, P., Shevchuk, I., 2020. Analysis of the flow conditions between the bottoms of the ship and of the waterway. Ocean Eng. 199. https://doi.org/10.1016/j.oceaneng.2020.107012

Celik, I.B., Ghia, U., Roache, P.J., Freitas, C.., 2008. Procedure for Estimation and Reporting of Uncertainty Due to Discretization in CFD Applications. J. Fluids Eng. 130, 078001. https://doi.org/10.1115/1.2960953

Chen, X., Zhu, R., Ma, C., Fan, J., 2016. Computations of linear and nonlinear ship waves by higher-order boundary element method. Ocean Eng. 114, 142-153. https://doi.org/10.1016/j.oceaneng.2016.01.016

Cole, S.L., 1987. Transient waves produced by a moving pressure distribution. Q. Appl. Math. 45, 51-58. https://doi.org/10.1090/qam/885167

Dam, K.T., Tanimoto, K., Fatimah, E., 2008. Investigation of ship waves in a narrow channel. J. Mar. Sci. Technol. 13, 223-230. https://doi.org/10.1007/s00773-008-0005-6

David, C.G., Roeber, V., Goseberg, N., Schlurmann, T., 2017. Generation and propagation of ship-borne waves - Solutions from a Boussinesq-type model. Coast. Eng. 127, 170-187. https://doi.org/10.1016/j.coastaleng.2017.07.001

Day, A.H., Clelland, D., Doctors, L.J., 2009. Unsteady finite-depth effects during resistance 
tests on a ship model in a towing tank. J. Mar. Sci. Technol. 14, 387-397. https://doi.org/10.1007/s00773-009-0057-2

Denner, F., Paré, G., Zaleski, S., 2017. Dispersion and viscous attenuation of capillary waves with finite amplitude. Eur. Phys. J. Spec. Top. 226, 1229-1238. https://doi.org/10.1140/epjst/e2016-60199-2

Doctors, L.J., 1975. The experimental wave resistance of an accelerating two-dimensional pressure distribution. J. Fluid Mech. 72, 513-527. https://doi.org/10.1017/S0022112075003114

Dorn, W.G.V., 1966. Boundary dissipation of oscillatory waves. J. Fluid Mech. 24, 769-779. https://doi.org/10.1017/S0022112066000995

Eca, L., Hoekstra, M., 2008. The numerical friction line. J. Mar. Sci. Technol. 13, 328-345. https://doi.org/10.1007/s00773-008-0018-1

Elsherbiny, K., Terziev, M., Tezdogan, T., Incecik, A., Kotb, M., 2020. Numerical and experimental study on hydrodynamic performance of ships advancing through different canals. Ocean Eng. 195. https://doi.org/10.1016/j.oceaneng.2019.106696

Elsherbiny, K., Terziev, M., Tezdogan, T., Incecik, A., Kotb, M., 2019a. Numerical and experimental study on hydrodynamic performance of ships advancing through different canals. Ocean Eng. 106696. https://doi.org/10.1016/j.oceaneng.2019.106696

Elsherbiny, K., Tezdogan, T., Kotb, M., Incecik, A., Day, S., 2019b. Experimental analysis of the squat of ships advancing through the New Suez Canal. Ocean Eng. 178, 331-344. https://doi.org/10.1016/j.oceaneng.2019.02.078

EMSA (European Maritime Safety Agency), 2019. Annual Overview of Marine Casualties and Incidents 2019.

EMSA (European Maritime Safety Agency), 2018. Annual Overview of Marine Casualties and Incidents 2018.

EMSA (European Maritime Safety Agency), 2017. Annual overview of marine casualties and incidents 2017.

EMSA (European Maritime Safety Agency), 2016. Annual overview of marine casualties and incidents 2016.

EMSA (European Maritime Safety Agency), 2015. Annual overview of marine casualties and incidents 2011-2015.

Ferziger, J.H., Peric, M., 2002. Computational Methods for Fluid Dynamics, Springer. https://doi.org/10.1016/S0898-1221(03)90046-0

Gourlay, T., 2003. Ship Squat In Water of Varying Depth. Int. J. Marit. Eng. 145, 12. https://doi.org/10.3940/rina.ijme.2003.a1.1031

Gourlay, T., 2001. The supercritical bore produced by a high-speed ship in a channel. J. Fluid Mech. 434, 399-409. https://doi.org/10.1017/S002211200100372X

Gourlay, T., Tuck, E.O., 2001. The maximum sinkage of a ship. J. Sh. Res. 45, 50-58.

Grue, J., 2017. Ship generated mini-tsunamis. J. Fluid Mech. 816, 142-166. https://doi.org/10.1017/jfm.2017.67 
Guo, J., 2002. Simple and explicit solution of wave dispersion equation. Coast. Eng. 45, 7174. https://doi.org/10.1016/S0378-3839(02)00039-X

Havelock, T., 1908. The Propagation of Groups of Waves in Dispersive Media, with Aplication to Waves on Water produced by a Travelling Disturbance 422-451. https://doi.org/10.1098/rspa.1933.0074

Hirt, C.. W., Nichols, B.. D., 1981. Volume of fluid (VOF) method for the dynamics of free boundaries. J. Comput. Phys. 39, 201-225. https://doi.org/10.1016/0021-9991(81)901455

Hunt, J.N., 1979. Direct solution of wave dispersion equation. J. Waterw. Port, Coastal, Ocean Eng. 106, 457-459.

Hunt, J.N., 1964. The viscous damping of gravity waves in shallow water. La Houille Blanche 685-691. https://doi.org/10.1051/lhb/1964038

Hur, V.M., 2019. Shallow water models with constant vorticity. Eur. J. Mech. B/Fluids 73, 170-179. https://doi.org/10.1016/j.euromechflu.2017.06.001

Jiang, T., Henn, R., Sharma, S.D., 2002. Wash waves generated by ships moving on fairways of varying topography. 24th Symp. Nav. Hydrodyn. 2, 8-13.

Johnson, J.W., 1957. Ship Waves in Navigation Channels. Coast. Eng. Proc. 1, 40. https://doi.org/10.9753/icce.v6.40

Katsis, C., Akylas, T.R., 1987. On the excitation of long nonlinear water waves by a moving pressure distribution. part 2. three dimensional effects. J. Fluid Mech. 177, 49-65. https://doi.org/10.1017/S0022112087000855

Katsis, C., Akylas, T.R., 1984. On the excitation of long nonlinear water waves by a moving pressure distribution. J. Fluid Mech. 177, 49-65. https://doi.org/10.1017/S0022112087000855

Keulegan, G.H., 1948. Gradual damping of solitary waves. J. Res. Natl. Bur. Stand. (1934). 40, 487. https://doi.org/10.6028/jres.040.041

Lamb, H., 1932. Hydrodynamics. Cambridge Univ. Press 6th revise, 262-264. https://doi.org/10.1017/CBO9781107415324.004

Lea, G.K., Feldman, J.P., 1972. Transcritical flow past slender ships, in: 9th Symposium on Naval Hydrodynamics. Washington DC, pp. 1527-1542.

Lee, B.W., Lee, C., 2019. Equation for ship wave crests in the entire range of water depths. Coast. Eng. 153, 103542. https://doi.org/10.1016/j.coastaleng.2019.103542

Liang, H., Chen, X., 2019. Viscous effects on the fundamental solution to ship waves. J. Fluid Mech. 879, 744-774. https://doi.org/10.1017/jfm.2019.698

Liu, H.W., Lin, P.Z., 2005. Discussion of "Wave transformation by two-dimensional bathymetric anomalies with sloped transitions" [Coast. Eng. 50 (2003) 61-84]. Coast. Eng. 52, 197-200. https://doi.org/10.1016/j.coastaleng.2004.11.002

Marshall, J.S., Naghdi, P.M., 1990. Wave reflection and transmission by steps and rectangular obstacles in channels of finite depth. Theor. Comput. Fluid Dyn. 1, 287-301. https://doi.org/10.1007/BF00271583 
Mathew, J., Akylas, T.R., 1990. On three-dimensional long water waves in a channel with sloping sidewalls. J. Fluid Mech. 215, 289-307. https://doi.org/10.1017/S0022112090002658

Miles, J.W., 1986. Stationary, transcritical channel flow. J. Fluid Mech. 162, 489-499. https://doi.org/10.1017/S0022112086002136

Muzaferija, S., Peric, M., 1999. Computation of free-surface ows using interface- tracking and interface-capturing methods, in: Chap. 2 in O. Mahrenholtz and M. Markiewicz (Eds.), Nonlinear Water Wave Interaction, Computational Mechanics Publications, WIT Press, Southampton.

Muzaferija, S., Peric, M., 1997. Computation of free-surface flows using the finite-volume method and moving grids. Numer. Heat Transf. Part B Fundam. 32, 369-384. https://doi.org/10.1080/10407799708915014

Newman, J.N., 1990. Numerical solutions of the water-wave dispersion relation. Appl. Ocean Res. 12, 14-18. https://doi.org/10.1016/S0141-1187(05)80013-6

Newman, J.N., 1965. Propagation of water waves over an infinite step. J. Fluid Mech. 23, 399415. https://doi.org/10.1017/S0022112065001453

Plotkin, A., 1977. Slender-ship shallow-water flow past a slowly varying bottom. J. Eng. Math. 11, 289-297. https://doi.org/10.1007/BF01537089

Plotkin, A., 1976. The flow due to a slender ship moving over a wavy wall in shallow water. J. Eng. Math. 10, 207-218. https://doi.org/10.1007/BF01535383

Roache, P.J., 1998. Verification of codes and calculations. AIAA J. 36, 696-702. https://doi.org/10.2514/2.457

Sharma, S.D., 1995. A Slender Ship Moving at a Near-Critical Speed in a Shallow Channel. J. Fluid Mech. 291, 263-285. https://doi.org/10.1017/S0022112095002692

Shevchuk, I., Böttner, C.U., Kornev, N., 2016. Numerical Analysis of the Flow in the Gap Between the Ship Hull and the Fairway Bottom in Extremely Shallow Water. Proc. 4th Int. Conf. Sh. Manoeuvring Shallow Confin. Water (MASHCON), 23 - 25 May 2016, Hamburg, Ger. 0, 37-42. https://doi.org/10.18451/978-3-939230-38-0

Siemens, 2018. Star-CCM+ User Guide version 13.04.

Soomere, T., 2007. Nonlinear components of ship wake waves. Appl. Mech. Rev. 60, 120138. https://doi.org/10.1115/1.2730847

Terziev, M., Tezdogan, T., Incecik, A., 2019. Application of eddy-viscosity turbulence models to problems in ship hydrodynamics. Ships Offshore Struct. https://doi.org/10.1080/17445302.2019.1661625

Terziev, M., Tezdogan, T., Oguz, E., Gourlay, T., Demirel, Y.K., Incecik, A., 2018. Numerical investigation of the behaviour and performance of ships advancing through restricted shallow waters. J. Fluids Struct. 76, 185-215. https://doi.org/10.1016/j.jfluidstructs.2017.10.003

Terziev, M., Zhao, G., Tezdogan, T., Yuan, Z., Incecik, A., 2020. Virtual Replica of a Towing Tank Experiment to Determine the Kelvin Half-Angle of a Ship in Restricted Water. J. Mar. Sci. Appl. 8, 1-24. https://doi.org/10.3390/jmse8040258 
Tezdogan, T., Demirel, Y.K., Kellett, P., Khorasanchi, M., Incecik, A., Turan, O., 2015. Fullscale unsteady RANS CFD simulations of ship behaviour and performance in head seas due to slow steaming. Ocean Eng. 97, 186-206. https://doi.org/10.1016/j.oceaneng.2015.01.011

Tezdogan, T., Incecik, A., Turan, O., 2016a. A numerical investigation of the squat and resistance of ships advancing through a canal using CFD. J. Mar. Sci. Technol. 21, 86101. https://doi.org/10.1007/s00773-015-0334-1

Tezdogan, T., Incecik, A., Turan, O., 2016b. Full-scale unsteady RANS simulations of vertical ship motions in shallow water. Ocean Eng. 123, 131-145. https://doi.org/10.1016/j.oceaneng.2016.06.047

Torsvik, T., 2009. Modelling of ship waves from high-speed vessels, in: Applied Wave Mathematics: Selected Topics in Solids, Fluids, and Mathematical Methods. Springer Berlin Heidelberg, pp. 229-263.

Torsvik, T., Dysthe, K., Pedersen, G., 2006. Influence of variable Froude number on waves generated by ships in shallow water. Phys. Fluids 18. https://doi.org/10.1063/1.2212988

Tuck, E.O., 1978. Hydrodynamic Problems of Ships in Restricted Waters. Annu. Rev. Fluid Mech. 10, 33-46.

Tuck, E.O., 1967. Sinkage and Trim in Shallow Water of Finite Width. Schiffstechnik 14, 9294.

Tuck, E.O., 1966. Shallow-Water Flows Past Slender Bodies. J. Fluid Mech. 26, 81-95. https://doi.org/10.1017/S0022112066001101

Wilcox, D.C., 2008. Formulation of the k-w Turbulence Model Revisited. AIAA J. 46, 28232838. https://doi.org/10.2514/1.36541

Wu, D.-M., Wu, T.Y., 1982. Three-Dimensional Nonlinear Long Waves Due to Moving Surface Pressure, in: 14th Symposium on Naval Hydrodynamics, Ann Arbor.

Yang, Q., Faltinsen, O.M., Zhao, R., 2001. Wash and Wave Resistance of Ships in Finite Water Depth. Pract. Des. Ships Other Float. Struct. 475-483. https://doi.org/10.1016/b978008043950-1/50060-0

Yuan, Z.M., 2014. Hydrodynamic interaction between ships travelling or stationary in shallow waters. Universtiy of Strathclyde.

Yuan, Z.M., Incecik, A., 2016. Investigation of side wall and ship model interaction, in: 2016 International Conference on Maritime Technology. pp. 1-10.

Yuan, Z.M., Zhang, X., Ji, C.Y., Jia, L., Wang, H., Incecik, A., 2018. Side wall effects on ship model testing in a towing tank. Ocean Eng. 147, 447-457. https://doi.org/10.1016/j.oceaneng.2017.10.042 